\title{
Resonance and cancellation phenomena in two-span continuous beams and its application to railway bridges
}

\author{
M.D. Martínez-Rodrigo ${ }^{\mathrm{a}, *}$, A. Andersson ${ }^{\mathrm{b}}$, C. Pacoste ${ }^{\mathrm{b}, \mathrm{c}}$, R. Karoumi $^{\mathrm{b}}$ \\ ${ }^{a}$ Universitat Jaume I, Department of Mechanical Engineering and Construction, Castellón, Spain \\ ${ }^{b}$ Division of Structural Engieering and Bridges, KTH Royal Institute of Technology, Stockholm, Sweden \\ ${ }^{c} E L U$ Konsult AB, Stockholm, Sweden
}

\begin{abstract}
The objective of this study is to evaluate the vibratory response of two-span continuous beams subjected to moving loads and, in particular, to investigate the maximum resonance and cancellation of resonance phenomena. The main practical interest is the evaluation of the maximum acceleration response in railway bridges, which is one of the most demanding Serviceability Limit States for traffic safety according to current regulations. Two-span continuous bridges, in their simplest version (i.e. uniform identical spans), present antisymmetric and symmetric modes with closely spaced natural frequencies, leading to a more involved dynamic behaviour than that of simply-supported bridges. First, the free vibration response of a BernoulliEuler two-span beam after the passage of a single load at constant speed is formulated analytically, and non-dimensional speeds leading to cancellation or maximum response in free vibration are obtained for each mode. Then, these conditions are equated to resonant speeds induced by equidistant load series, and span length-to-characteristic distance ratios causing cancelled out resonances, or remarkably prominent ones, are obtained. Based on the previous derivations, a methodology for detecting which could be the most aggressive trains for a particular structure based on pure geometrical considerations is discussed. Finally, the applicability of the theoretical derivations is shown through the numerical analysis of two real bridges belonging to the Swedish railway network.
\end{abstract}

Keywords: Railway bridges, two-span continuous beams, resonance, cancellation, moving loads.

\section{Introduction}

The progressive increase in operating speeds on railway lines constitutes a challenge for administrations, rolling stock manufacturers and engineers as vibration levels that are admissible for passengers, vehicles, infrastructures and surrounding structures must be guaranteed. In this regard, railway bridges have received 5 considerable attention during the last decades. The periodic excitation caused by the axle loads crossing a

\footnotetext{
*Corresponding author.

Email address: mrodrigo@uji.es (M.D. Martínez-Rodrigo)
} 
bridge or a viaduct at constant speed may induce significant levels of vertical oscillations on the deck, which can lead to adverse consequences such as ballast deconsolidation, track misalignment, passenger discomfort or even wheel-rail contact loss and associated risks [1], [2, 3].

Among the longitudinal typologies of railway viaducts, both bridges with continuous decks resting on multiple supports and bridges composed of simply-supported (SS) spans coexist. The former, common in countries such as Spain, Sweden or France, are structurally more efficient and able to transmit the horizontal break and acceleration forces to the ground with the collaborative action of the substructure elements. The latter, frequently found in countries like Germany or China, may be constructed in a rather systematic way, allow possible prefabrication, partial replacement of the SS decks and facilitate continuous rails. Nevertheless, the higher number of joints and supporting devices increases the maintenance costs and these structures are usually appropriate only when piers have a limited height [4, [5]. Simply-supported bridges have received considerably more attention than continuous structures. This work is devoted to twospan continuous bridges, as the simultaneous contribution to the transverse vibrations of antisymmetric and symmetric modes constitutes a more complex problem and these structures may still experience important amplifications under railway traffic [6], [7].

The basic phenomenon governing the level of vibrations induced in a bridge by a railway convoy is the amplitude of the free vibrations that each axle leaves on the structure after its passage, as these free vibration waves accumulate and may add in phase for certain speeds. Depending on the ratio between the travelling time of the load and the natural period of the structure, the amplitude of the free vibrations in that particular mode may be maximum or may be cancelled out, implying that the structure will remain at rest under certain ideal conditions (i.e. in the absence of structural damping). This basic problem has been analysed in detail by authors such as Yang et al. [8, [9], Savin [10, Museros et al. [11] and Kumar et al. 12] for simply- and elastically-supported beams. The interest of knowing these conditions a priori is that when resonance, caused by series of loads, takes place at a maximum free vibration velocity or, on the contrary, close to a cancellation condition, the train will induce either a very prominent response or an almost imperceptible one, respectively.

The problem of a continuous uniform beam with two equal spans traversed by a concentrated force moving at constant speed was first solved by Ayre et al. [13, who described the moving load as a series of pulsating forces. The early works on the problem of moving loads acting on simple structures is described in detail in the classic reference by Frýba [14. In the 1990s, Zheng et al. [15] and Cheung et al. [16] analysed the vibrations of multi-span beams subjected to moving forces and oscillators, respectively, using modified beam vibration functions as the assumed modes. The authors showed fast convergence of the method with a small number of unknowns when compared to Finite Element (FE) solutions. Yau [7 investigated the effect of the number of spans in the dynamic response of multi-span uniform beams subjected to train loads. 
peaks as the speed increases, but also in a reduction in the impact response. This is attributed to the transmission of the vibration energy to the neighbouring spans and to the higher restraining effect of the supports on the transverse displacement. Dugush and Eisenberger [17] obtained the exact solution of the moving load problem on multi-span non-uniform beams for any polynomial variation in the cross-section properties using the exact element method. Johansson et al. [18 derived a closed-form exact solution for evaluating the dynamic behaviour of a general multi-span Bernoulli-Euler (BE) beam under constant moving loads, considering stepped sections and elastic boundary conditions.

Publications devoted to resonance or its cancellation in two-span beams or bridges are rather scarce and recent. Yau [7] investigated the impact response of continuous beams and, considering moving loads, detected multiple resonant peaks due to the coincidence of the excitation frequency with the beam frequencies. Kwark et al. 19 presented one of the first experimental studies on the dynamic behaviour of a two-span continuous bridge under the circulation of Korean High-Speed trains along with FE predictions including vehicle-bridge interaction. Resonances of only the fundamental antisymmetric mode were captured for the particular design speed. The authors focused on the effect of damping and vehicle-structure interaction on the transverse displacement response. Wang et al. 20] analysed the resonant response of two-span bridges modelled as BE uniform beams subjected to trains of moving oscillators. The authors focused on the vertical response in terms of displacements, and on the appearance of two critical speeds causing resonance associated to the first antisymmetric and first symmetric modes of vibration. Wang et al. [6] numerically investigated the vertical acceleration of two-span continuous bridges with long spans (40 and $45 \mathrm{~m}$ ) and uniform cross-sections under the action of High-Speed trains, modelled as equally spaced 2-degrees-of-freedom mass-spring-damper units. This is one of the few publications to focus on the acceleration response of the bridge rather than on the displacement. The authors concluded that the resonant acceleration response in bridge and vehicle may be amplified to a fairly high degree, especially for the first two resonant speeds that may fall within the range of operating speeds of High-Speed trains. Moreover, due to the presence of sub-resonant speeds of higher modes, the maximum acceleration may occur at sections other than mid-span.

In the authors' opinion, what it is presented herein is useful, novel and contributes to the knowledge on the dynamic performance of railway bridges as (i) it provides a comprehensive study on the cancellation and maximisation of resonance in two-span continuous bridges based of the free vibration response of the structures; (ii) previous publications generally address specific structures and trains and, in the opinion of the authors, the problem should be formulated non-dimensionally, in order to reach general conclusions; (iii) most of the previous contributions focus on the displacement response of the bridge, generally governed by only a few modes [21-22], and not on the acceleration, which is a far more restrictive Serviceability Limit State for these structures according to current regulations 23]; and (iv) being able to predict velocities leading to maximum free vibration or cancellation is of practical interest as not only the most and least aggressive trains may be detected for a particular structure but, in addition, this information could also be 
useful when planning experimental campaigns on bridges with the aim of identifying amplitude-dependent magnitudes (e.g. modal damping).

The objectives of this study are to (i) investigate analytically the problem of free vibrations in two-span continuous beams; (ii) verify whether maximum free vibration and cancellation conditions take place and, if so, to determine their value for any longitudinal bending mode and for any structure; (iii) obtain geometrical ratios leading to maximum resonance and cancellation of it for symmetric and antisymmetric modes and to prove their applicability when ideal conditions are not met; and (iv) apply the former theoretical derivations to the application of two-span bridges under High-Speed traffic by proposing a methodology to detect which could be the most and least aggressive trains for a particular design speed of the line, and what kind of resonance (order and mode) is responsible for it.

The contents of the manuscript are organised as follows. In section 2 the free vibration response of a BE two-span continuous beam under a single moving load is formulated analytically, and maximum free vibration and cancellation non-dimensional speeds are presented and obtained for each mode. In section 3 the excitation caused by trains of equidistant loads is considered and length-to-characteristic distance ratios are derived leading to maximum or cancelled different order resonances of any mode of the beam. In section 4 a methodology is proposed so as to be able to compare the effect of different trains on the maximum acceleration response of the bridge admitting a maximum operational speed for the line. Finally, two bridges from the Swedish railway network are evaluated in order to show the applicability of the former theoretical derivations. Conclusions are presented in section 5.

The derivations and conclusions presented herein are limited to the effect of the geometry of the trains on the bridge response. Additional phenomena conforming the train-induced vibration problem may affect the maximum acceleration response, such as vehicle-track-bridge $(24,25,26])$ or soil-structure interaction $(27,, 28])$. The influence of these effects on the maximum response of the bridge may or may not be relevant depending on the level of coupling between the subsystems. This study pretends to constitute a firm theoretical base on which additional interaction effects, as those previously mentioned which entail an important level of uncertainty, may be evaluated in subsequent investigations.

\section{Free vibrations of a two-span continuous beam under a single moving load}

\subsection{Amplitude of free vibrations in undamped case}

The partial differential equation governing the undamped transverse vibrations of a $\mathrm{BE}$ beam, neglecting shear deformation and rotatory inertia, traversed by a constant-valued load $P$ moving at constant speed (see Fig. 1(a)) is given by

$$
\rho A(x) \frac{\partial^{2} w(x, t)}{\partial t^{2}}+\frac{\partial^{2}}{\partial x^{2}} E I(x) \frac{\partial^{2} w(x, t)}{\partial x^{2}}=-P \delta(x-V t)\left[H(t)-H\left(t-\frac{2 L}{V}\right)\right]
$$


where $w(x)$ is the transverse displacement of a generic section $x$ at time $t, \rho A(x)$ is the mass per unit of length of the beam and $E I(x)$ represents the cross-section bending stiffness. In Eq. 1, $\delta$ and $H$ stand for Dirac Delta and Heaviside unit functions, respectively. The solution to Eq. 1 may be expressed as a linear combination of the beam normal modes of vibration $\phi_{i}(x)$ as per

$$
w(x, t)=\sum_{i=1}^{\infty} \xi_{i}(t) \phi_{i}(x)
$$

For the simplest case of a two-equal-span uniform beam with total length $2 L$ (see Fig. 1(a)), applying appropriate boundary conditions and performing a free vibration analysis [29], the analytical normal modes are obtained for antisymmetric and symmetric modes, $\phi_{i}^{a}$ and $\phi_{i}^{s}$, respectively, and can be expressed as

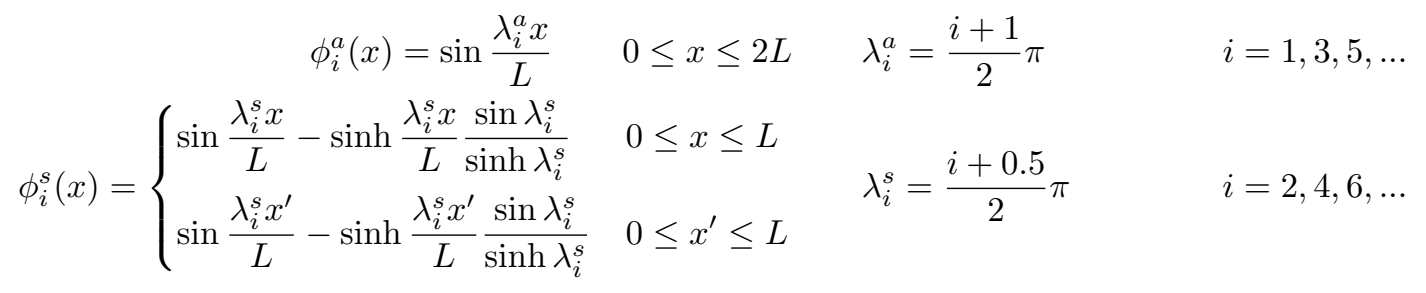

where $x^{\prime}=2 L-x$. In Eqs. 3a $3 \mathrm{~b}$ and in what follows, superscripts $a$ and $s$ are used to differentiate antisymmetric and symmetric modes when considered necessary. $\lambda_{i}^{a}$ and $\lambda_{i}^{s}$ are the roots of the frequency equation (Eq. 4a) and are related to the circular frequencies as per Eq. $4 \mathrm{~b}$

$$
\begin{array}{r}
\sin (\lambda)(\cos (\lambda)-\operatorname{coth}(\lambda) \sin (\lambda))=0 \quad \Rightarrow \quad \lambda_{i}^{a}, \lambda_{i}^{s} \\
\omega_{i}^{a}=\left(\frac{\lambda_{i}^{a}}{L}\right)^{2} \sqrt{\frac{E I}{\rho A}} \quad \omega_{i}^{s}=\left(\frac{\lambda_{i}^{s}}{L}\right)^{2} \sqrt{\frac{E I}{\rho A}}
\end{array}
$$

In Figs. 1(c)-(d) the first three antisymmetric and first three symmetric modes are represented for equal maximum displacement normalisation. Antisymmetric and symmetric modes alternate with the frequency number, and each modal frequency increases in relation to the fundamental one according to the factors

$$
f_{i}=f_{1}^{a} \cdot\{\mathbf{1}, 1.56, \mathbf{4}, 5.06, \mathbf{9}, 10.56, \mathbf{1 6}, 18.06, \ldots\}
$$

where bold numbers correspond to antisymmetric modes frequencies.

By substitution of Eqs. 3a, 3b and 2 into 1, multiplication by the $n$-th mode, integration along the beam length, and in virtue of the orthogonality condition of the modes, the uncoupled equation governing the $n$-th modal amplitude is obtained:

$$
\ddot{\xi}_{n}(t)+\omega_{n}^{2} \xi_{n}(t)=-\frac{P}{M_{n}} \phi_{n}(x=V t) \quad M_{n}=\int_{0}^{2 L} \rho A \phi_{n}(x)^{2} d x=\rho A L
$$


(a)
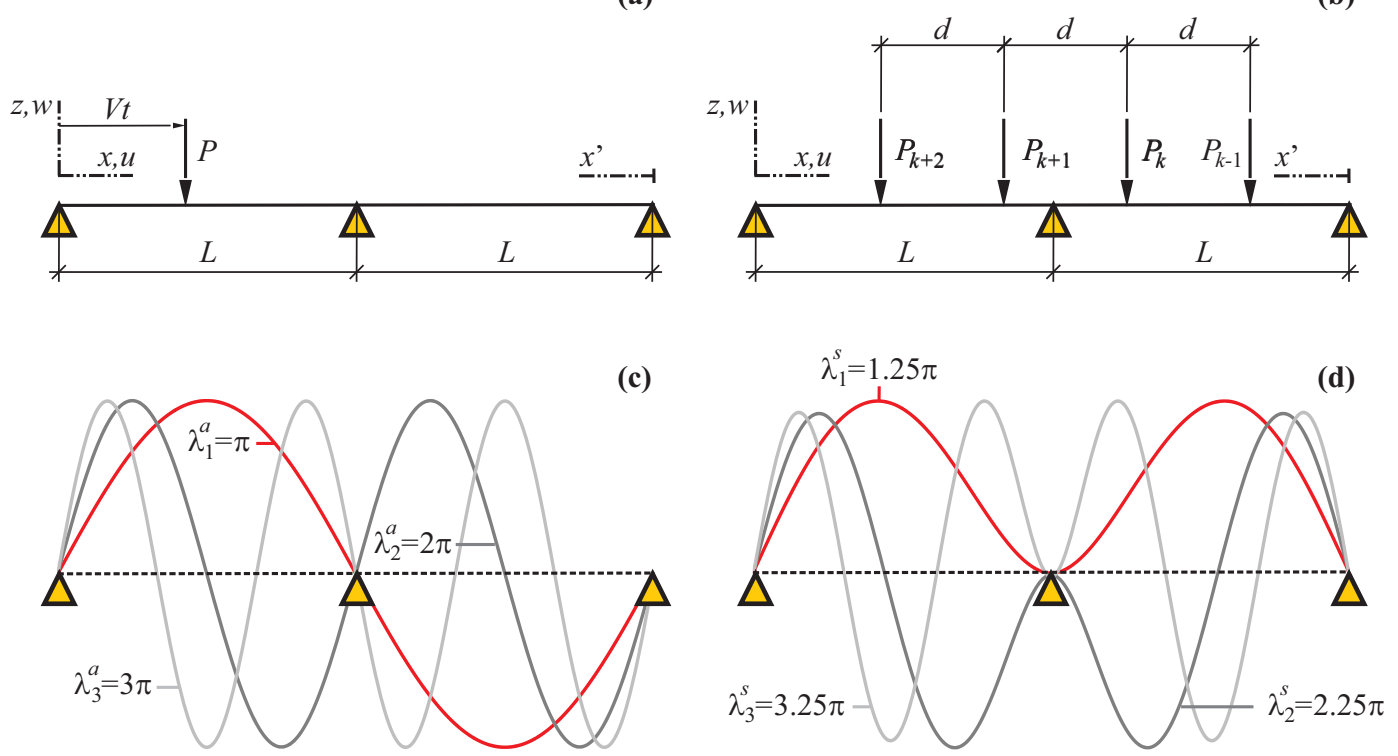

Figure 1: Two-span uniform beam under moving loads travelling at constant speed (a)-(b). First three (c) antisymmetric and (d) symmetric normal modes.

In order to determine the amplitude of the free vibrations once the load $P$ leaves the structure, the previous equation and its first derivative are solved by convolution for the particular instant $t=2 L / V$

$$
\xi_{n}\left(t=\frac{2 L}{V}\right)=\frac{-P}{\omega_{n} M_{n}} \int_{0}^{\frac{2 L}{V}} \phi_{n}(V \tau) \sin \left[\omega_{n}(t-\tau)\right] d \tau \quad \dot{\xi}_{n}\left(t=\frac{2 L}{V}\right)=\left.\frac{d \xi_{n}(t)}{d t}\right|_{t=\frac{2 L}{V}}
$$

Finally, the amplitude of the free vibrations in each mode is obtained and non-dimensionalised by the modal static displacement as per Eq. 8. By doing so, the so-called normalised amplitude of the free vibrations, $R_{n}$, can be obtained for antisymmetric and symmetric modes in terms of a single non-dimensional velocity, $K_{n}$. This speed parameter has also been used by previous authors 9 . In Eq. 9 the particular closed form expression for the antisymmetric modes is provided. For the symmetric case the analytical expression is rather involved and it is not included for the sake of conciseness. Nonetheless, $R_{n}$ may be computed for any mode either analytically or by numerical evaluation of Eq. 7. Notice that the amplitude of the free vibrations does not decay, as damping is not considered in this section.

$$
\begin{array}{cc}
R_{n}=\frac{\omega_{n}^{2} M_{n}}{-P} \sqrt{\frac{\left.\dot{\xi}_{n}^{2}\right|_{t=\frac{2 L}{V}}}{\omega_{n}^{2}}+\left.\xi_{n}^{2}\right|_{t=\frac{2 L}{V}}} \Rightarrow & R_{n}=f\left(K_{n}=\frac{\lambda_{n} V}{\omega_{n} L}\right) \\
R_{n}=\frac{\sqrt{2} K_{n}}{1-K_{n}^{2}} \sqrt{1-\cos \left[\frac{(1+n) \pi}{K_{n}}\right]} & n=1,3,5, \ldots
\end{array}
$$


In Figures 2(a) and 2(b), the evolution of $R_{n}$ is presented versus the speed parameter corresponding to the fundamental mode, $K_{1}$, for the first antisymmetric $(n=1)$ and the first symmetric $(n=2)$ modes of the two-span uniform beam, respectively. Fig. 2(c) also represents $R_{n}$ versus $K_{1}$ but for the first three antisymmetric modes (i.e. $n=1,3,5)$. As both $R_{n}$ and $K_{n}$ are non-dimensional, these representations and the conclusions derived hereafter are applicable to any two-equal-span uniform beam. Representing the response with respect to the same speed parameter $\left(K_{1}\right.$ instead of $\left.K_{n}\right)$ allows direct comparison in terms of the speed $V$ for the different modal responses. The relation between any modal speed parameter $K_{n}$ and the one referred to the fundamental mode can easily be deduced for antisymmetric and symmetric modes as

$$
K_{n}= \begin{cases}\frac{2}{n+1} K_{1} & n=1,3,5, \ldots \\ \frac{2}{n+0.5} K_{1} & n=2,4,6, \ldots\end{cases}
$$

From the analysis of Fig. 2 the following can be concluded:

- Depending on the travelling speed, the amplitude of the free vibrations that a particular beam undergoes in a certain mode once the load leaves the structure can be maximum or cancelled out, taking into account that no damping is present in the system. The speed parameters for these cancellation and maximum free vibration conditions, $K_{1}^{c i}$ and $K_{1}^{m i}$, can be obtained analytically and are pointed out in Figures 2(a)-(b) for the first antisymmetric $(n=1)$ and the first symmetric $(n=2)$ modes, respectively. Notice that index $i$ refers to a particular cancellation or maximum free vibration event, and that $i=1$ corresponds to the event taking place at the highest velocity.

- The velocities cancelling out the free vibrations of the fundamental mode also cancel out the response of the remaining antisymmetric modes (see Fig. 2(c)). This does not occur among the symmetric modes or among these and the fundamental mode.

- When damping is present and for moderate levels of it, like those usually identified in railway bridges [1], $R_{n}$ presents a similar evolution in terms of the speed parameter $K_{n}$. The main differences in the damped case are that (i) amplitudes at local maxima are lower, and that (ii) the response is not completely cancelled out at cancellation speeds, although the response is remarkably low. The effect of damping will be accounted for in sections 3 and 4.

Finally, it is of interest to note that, due to the selected normalisation and to the relation between displacement and acceleration amplitudes in the undamped case, the dimensional vertical displacement and acceleration amplitudes in a particular section of the two-span beam in the free vibration phase induced by a load $P$ is related to $R_{n}$ according to

$$
\bar{w}_{n}^{\text {free }}(x)=R_{n} \cdot \frac{P}{\omega_{n}^{2} \rho A L} \cdot \phi_{n}(x) \quad \bar{a}_{n}^{\text {free }}(x)=R_{n} \cdot \frac{P}{\rho A L} \cdot \phi_{n}(x)
$$



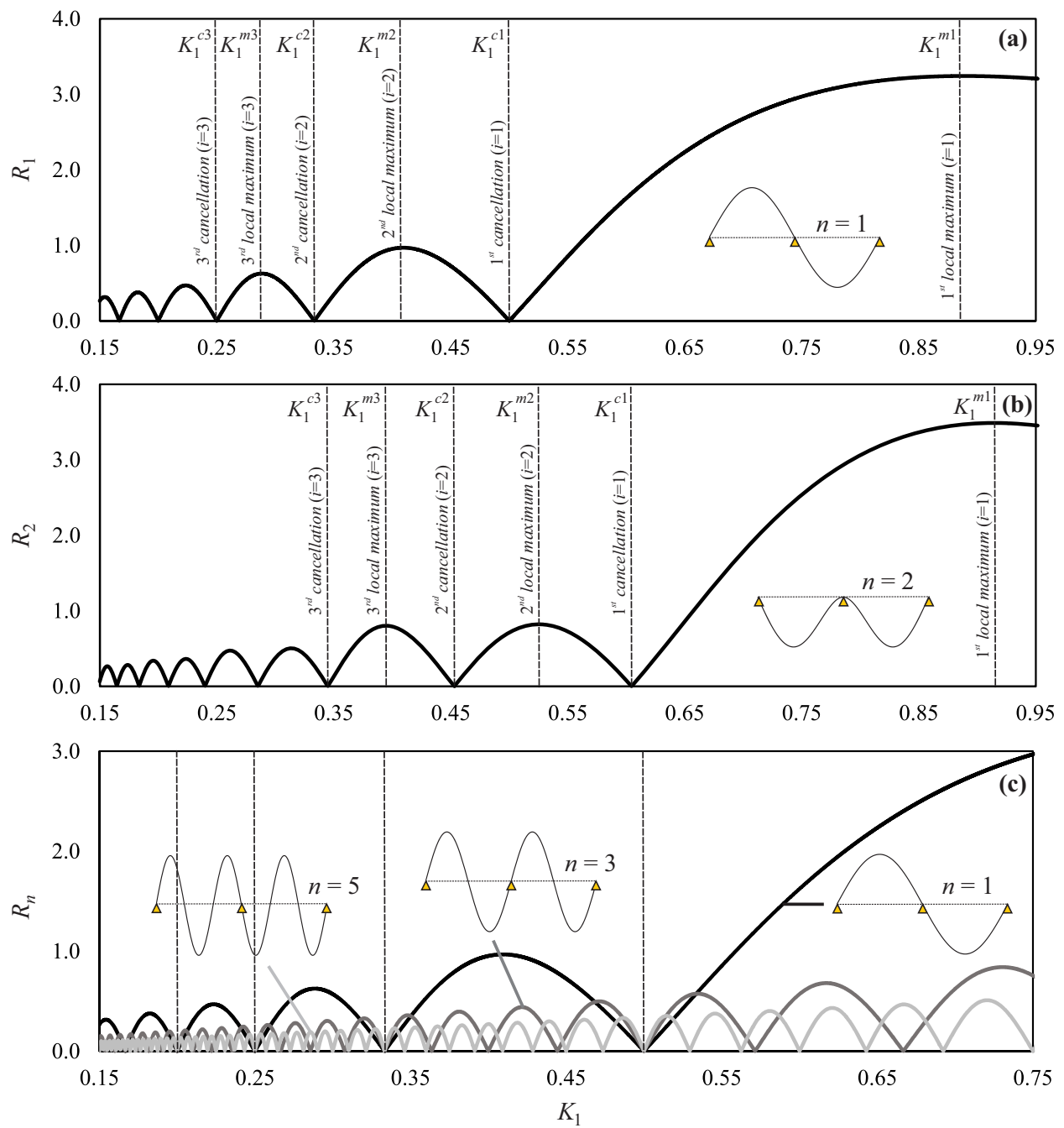

Figure 2: $R_{n}$ for (a) first antisymmetric mode, (b) first symmetric mode, and (b) first three antisymmetric modes vs. $K_{1}$.

From Eq. 11 it is seen that the relative differences between $R_{n}$ values in Fig. 2 are proportional to the relative differences between the acceleration amplitudes in free vibration in a certain mode and in a particular section.

\subsection{Cancellation and maximum free vibration speeds}

From the solution of $R_{n}=f\left(K_{n}\right)$ (Eq. 8) cancellation and maximum free vibration non-dimensional speeds may be calculated as

$$
R_{n}\left(K_{n}\right)=0 \Rightarrow K_{n}^{c i} \quad \frac{\partial R_{n}\left(K_{n}\right)}{\partial K_{n}}=0 \Rightarrow K_{n}^{m i} \quad i=1,2,3, \ldots
$$

From now on, $K_{n}^{c i}$ and $K_{n}^{m i}$ stand for the speed parameters leading to the $i$-th cancellation and $i$-th maximum free vibration conditions in mode $n$, respectively. In Table 1 the values for the first four cancellation and 
maximum free vibration speeds are included for the first two antisymmetric and the first two symmetric modes. As should be expected, the values for the fundamental mode coincide with those of the SS beam for the second bending mode if $L$ is the span length [11].

\begin{tabular}{|c|c|c|c|c|c|c|c|c|}
\hline \multirow{2}{*}{$n$} & \multicolumn{4}{|l|}{$K_{n}^{c i}$} & \multicolumn{4}{|l|}{$K_{n}^{m i}$} \\
\hline & $i=1$ & $i=2$ & $i=3$ & $i=4$ & $i=1$ & $i=2$ & $i=3$ & $i=4$ \\
\hline 1 & 0.5000 & 0.3333 & 0.2500 & 0.200 & 0.8883 & 0.4094 & 0.2886 & 0.2235 \\
\hline 3 & 0.6667 & 0.5000 & 0.4000 & 0.3333 & 0.9653 & 0.5812 & 0.4478 & 0.3652 \\
\hline 2 & 0.4835 & 0.3624 & 0.2758 & 0.2282 & 0.7312 & 0.4202 & 0.3157 & 0.2509 \\
\hline 4 & 0.6201 & 0.5107 & 0.4044 & 0.3488 & 0.8409 & 0.5625 & 0.4542 & 0.3758 \\
\hline
\end{tabular}

Table 1: Values for the first four cancellation and maximum free vibration speed parameters for the first two antisymmetric $(n=1,3)$ and first two symmetric $(n=2,4)$ modes.

As the main practical interest of this research is the evaluation of railway-induced vibrations in two-span bridges, it is worth mentioning that a realistic upper limit for $K_{1}$, which will always present the highest value of $K_{n}$ according to Eq. 10, can be estimated. Admitting an average bridge fundamental frequency in terms of the span length as per [30, considering a maximum operational speed of $500 \mathrm{~km} / \mathrm{h}$ and a minimum span length of $15 \mathrm{~m}$, a maximum value $K_{1} \simeq 0.5$ is obtained. Therefore, the overall first maximum corresponding to $i=1$ will never be reached in a realistic situation.

\section{Forced vibrations under equidistant loads: maximum resonance and cancellation}

\subsection{Span length-to-characteristic distance ratios for maximum resonance and cancellation of resonance}

In this section the dynamic response of the two-span beam is investigated under trains of equidistant loads travelling at constant speed $V$ (see Fig. 1(b)). When the time interval between the passage of two consecutive loads is a multiple of one of the beam natural periods, resonance is induced. At resonance, the free vibrations left by every single load add in phase. Therefore, depending on the amplitude of the free vibrations studied in section 2, resonance could result in a remarkably amplified response (if the resonant speed coincides with a maximum free vibration speed) or may not even be perceptible (if it coincides with or is close to a cancellation condition). Ideally, a train of equidistant loads with characteristic distance $d$ induces a $j$-th order resonance of the $n$-th mode when travelling at $V_{n j}^{r}$, as per Eq. 13, [9]. Moreover, the resonant speed may be expressed non-dimensionally, according to the speed parameter definition in Eq. 8 ,

as

$$
V_{n j}^{r}=\frac{d f_{n}}{j} \Rightarrow K_{n j}^{r}=\frac{\lambda_{n}}{\omega_{n} L} \frac{d f_{n}}{j}
$$


By equating Eq. 13 to the cancellation or maximum free vibration speed parameters included in Table 1. for any mode $n$ and cancellation or maximum event $i, L / d$ ratios leading to the cancellation or maximum of the $j$-th resonance of the $n$-th mode of the two-span continuous beam are obtained according to

$$
\begin{array}{r}
K_{n j}^{r}=K_{n}^{c i} \Rightarrow\left(\frac{L}{d}\right)_{n j}^{c i}=\frac{\lambda_{n}}{2 \pi j} \frac{1}{K_{n}^{c i}} \quad K_{n j}^{r}=K_{n}^{m i} \Rightarrow\left(\frac{L}{d}\right)_{n j}^{m i}=\frac{\lambda_{n}}{2 \pi j} \frac{1}{K_{n}^{m i}} \\
\text { where } \quad \frac{\lambda_{n}}{2 \pi j}= \begin{cases}\frac{n+1}{4 j} & n=1,3,5, \ldots \\
\frac{n+0.5}{4 j} & n=2,4,6, \ldots\end{cases}
\end{array}
$$
and first symmetric modes, which will have the highest participation in the transverse acceleration, as will be shown later on. Ratios for higher modes could also be obtained analytically by applying Eq. 14a. These ratios are valid for any beam or bridge due to the non-dimensional nature of the formulation.

\begin{tabular}{llllllllll}
\hline & $(L / d)_{1 j}^{c i}$ & & & & & $(L / d)_{1 j}^{m i}$ \\
\cline { 2 - 3 } \cline { 7 - 9 } & $i=1$ & $i=2$ & $i=3$ & $i=4$ & & $i=1$ & $i=2$ & $i=3$ & $i=4$ \\
\hline 1 & 1.000 & 1.500 & 2.000 & 2.500 & & 0.563 & 1.221 & 1.733 & 2.238 \\
2 & 0.500 & 0.750 & 1.000 & 1.250 & & 0.281 & 0.611 & 0.866 & 1.119 \\
3 & 0.333 & 0.500 & 0.667 & 0.833 & & 0.188 & 0.407 & 0.578 & 0.746 \\
4 & 0.250 & 0.375 & 0.500 & 0.625 & & 0.141 & 0.305 & 0.433 & 0.559 \\
\hline
\end{tabular}

\begin{tabular}{|c|c|c|c|c|c|c|c|c|}
\hline \multirow{2}{*}{$j$} & \multicolumn{4}{|c|}{$(L / d)_{2 j}^{c i}$} & \multicolumn{4}{|c|}{$(L / d)_{2 j}^{m i}$} \\
\hline & $i=1$ & $i=2$ & $i=3$ & $i=4$ & $i=1$ & $i=2$ & $i=3$ & $i=4$ \\
\hline 1 & 1.293 & 1.725 & 2.266 & 2.739 & 0.855 & 1.487 & 1.980 & 2.491 \\
\hline 2 & 0.646 & 0.862 & 1.133 & 1.369 & 0.427 & 0.744 & 0.990 & 1.246 \\
\hline 3 & 0.431 & 0.575 & 0.755 & 0.913 & 0.285 & 0.496 & 0.660 & 0.830 \\
\hline 4 & 0.323 & 0.431 & 0.567 & 0.685 & 0.214 & 0.372 & 0.495 & 0.623 \\
\hline
\end{tabular}

Table 2: $L / d$ ratios leading to cancellation of resonance and maximum resonance of the first mode (antisymmetric).

Table 3: $L / d$ ratios leading to cancellation of resonance and maximum resonance of the second mode (symmetric).

\subsection{Maximum displacement and acceleration numerical response: contour plots.}

In the previous sections, the cancellation and maximum free vibration phenomena for two-span B-E continuous beams have been formulated analytically. In addition, the $L / d$ ratios for cancellation of resonance and maximum resonance have been depicted when series of equidistant loads traverse the beam. In what 
follows, these phenomena are exemplified through numerical parametric analyses including the presence of structural damping and considering the simultaneous contribution of several modes of vibration in the computation of the transverse response.

A particular beam is selected with the properties of an existing railway bridge that will be analysed in section 4. In particular $L=23.5 \mathrm{~m}, E I=7.14 \cdot 10^{10} \mathrm{Nm}^{2}, \rho A=23010 \mathrm{~kg} / \mathrm{m}$ and modal damping $\zeta_{n}=1 \%$. The bridge vertical response is obtained in the time domain by numerical integration applying the Newmark- $\beta$ linear acceleration algorithm 31 under the circulation of artificial trains of equidistant loads. The analytical expressions for the normal modes (Eqs. 3a $3 \mathrm{~b}$ are used to represent the deformation of the beam and, therefore, no spatial discretisation is needed. The vertical response of the bridge is obtained under the circulation of 201 independent trains each composed of 25 loads of $210 \mathrm{kN}$ separated by a distance $d$. Characteristic distances, $d$, are selected so that the range $(L / d) \in[0.5,2.5]$ is covered using a fine increment $\Delta(L / d)=0.01$. For each of the trains, the maximum displacement and acceleration are obtained at different sections of the beam in a range of circulating velocities such that the first three resonances $(j=1,2,3)$ of the first and of the second modes are excited. In particular, the minimum, maximum and increment of the velocity analysed for each particular train are obtained from the conditions

$$
\left(\frac{V}{f_{1} d}\right)_{\min }=0.1 \quad\left(\frac{V}{f_{1} d}\right)_{\max }=2.0 \quad \Delta\left(\frac{V}{f_{1} d}\right)=0.005
$$

Finally, the maximum transverse response is computed in two scenarios: considering the contribution of the first two modes, and considering the contribution of the first six modes of vibration (modes with natural frequencies up to $60 \mathrm{~Hz}$ ). The natural frequencies of the modes in the particular case under study are the following:

$$
f_{n}=\{5.01,20.04,45.09\}[\mathrm{Hz}] \quad n=1,3,5 \quad f_{n}=\{7.83,25.37,52.92\}[\mathrm{Hz}] \quad n=2,4,6
$$

In Fig. 3, contour plots of the maximum transverse response of the beam in absolute value due to the contribution of the first two modes $(N=2)$ are included. Each point in the contour representation corresponds to a particular train, defined by its characteristic distance $d$, and a particular circulating velocity $V$ of that train. A similar form of representation was used as well in the past by Yang et al. 9] and by Yau [7 for different types of beams. Figures (a) and (c) correspond to the mid-span of the first span, and figures (b) and (d) to the central section of the second span. Maximum displacements are included in the first row, while maximum accelerations are represented in the second row. The maximum response for each train and velocity is plotted in terms of two ratios: $L / d$ and the speed $V$ divided by the product of the fundamental frequency times the characteristic distance. This representation is not only non-dimensional, but it also facilitates the visualisation of a particular mode resonant order, as the corresponding peaks appear vertically aligned for each value of $j$. In all the plots a series of maxima appear at $V / f_{1} d=1,0.5,0.333, \ldots$ for different $L / d$ values corresponding to resonances of the fundamental antisymmetric mode for $j=1,2,3, \ldots$ 
Series of peaks are also visible in the vicinity of $V / f_{1} d=1.56,0.78,0.52, \ldots$, coinciding with resonances of the second mode (first symmetric mode), as well for $j=1,2,3, \ldots$ These values are consistent with the fact that $f_{2}=1.56 f_{1}$ (Eq. 5).
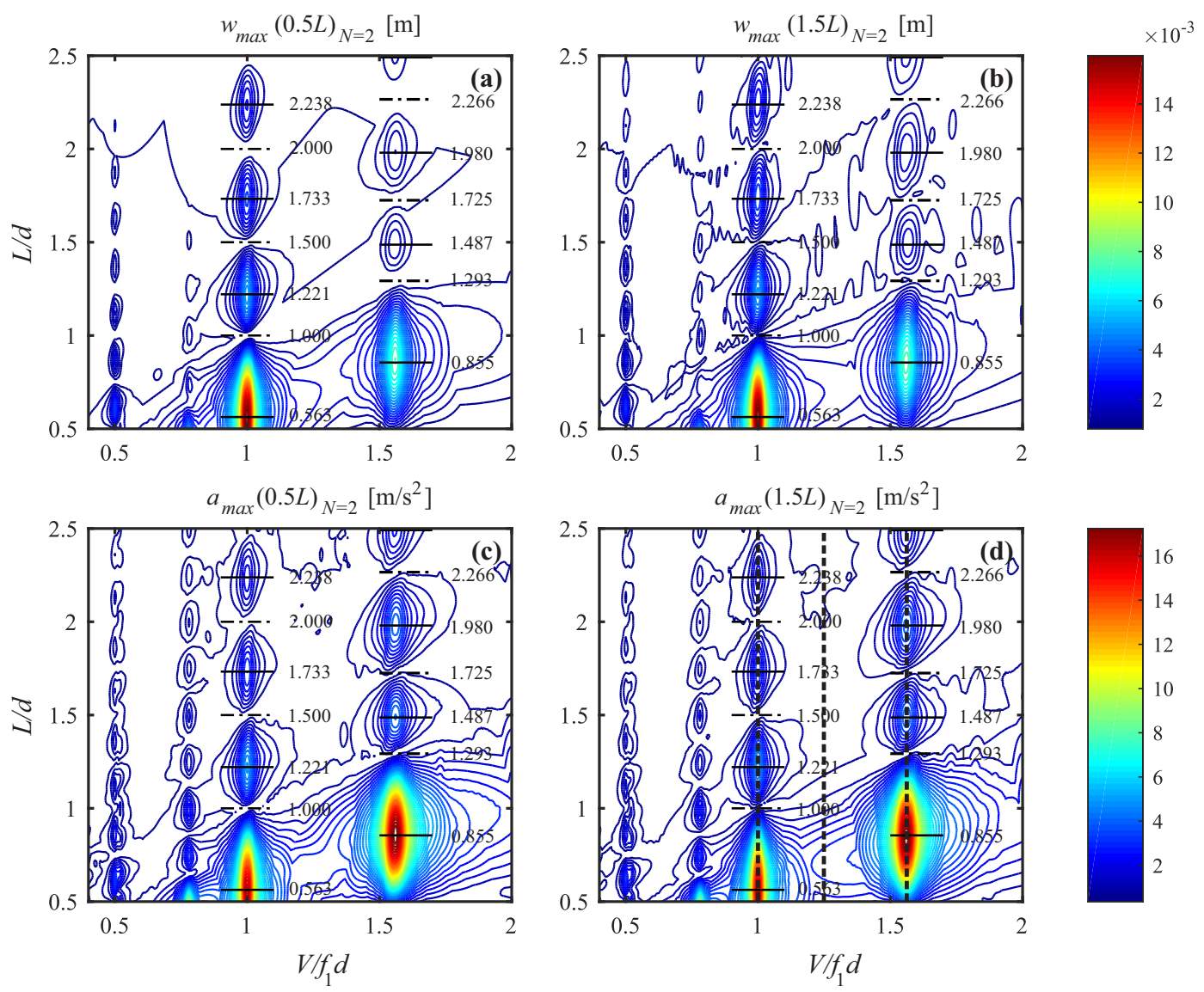

Figure 3: Maximum displacement and acceleration at $x=0.5 L$ and $x=1.5 L$ contour plots for $N=2$.

At resonance, as $L / d$ increases maximum resonance peaks and cancelled resonances take place alternatively. In Fig. 4(a) maximum acceleration envelopes at $x=1.5 \mathrm{~L}$ are plotted for $V / f_{1} d=1,1.56$ and 1.25 (see vertical dashed lines in Fig. $3(\mathrm{~d})$ ). The first two correspond to first resonances of the fundamental and the second mode. The latter is not a resonant condition for either of the two modes. It is worth noting that as $L / d$ increases, the amplification at resonance reduces, since shorter characteristic distance trains excite the same resonance at a lower velocity, and lower velocities imply lower amplitude local maxima of the free vibration response, as shown in section 2. Fig. 4(b) shows the same maximum acceleration curves but computed with 6 modes. It can be seen how the contribution of higher modes is not very relevant for these speed ratios, as will be shown in more detail in what follows.

The actual value of the maximum response in Fig. 3 is not relevant for this study, as no upper limit 
for the train speed is considered. In all plots and for the first resonance order $(j=1)$ of the first two modes, corresponding to $V / f_{1} d=1$ and $1.56, L / d$ analytical ratios for maximum resonance and cancellation of resonance have been highlighted in thick and discontinuous horizontal segments along with the specific value, which is that included in Tables 2 and 3 . The analytical predictions are accurate, even though the two modes contribute simultaneously to the total response and despite the presence of structural damping. It is also worth mentioning that these ratios predict cancelled and maximum resonances adequately both in the displacement and in the acceleration response.

$$
a_{\max }(1.5 L)_{N=2}\left[\mathrm{~m} / \mathrm{s}^{2}\right]
$$

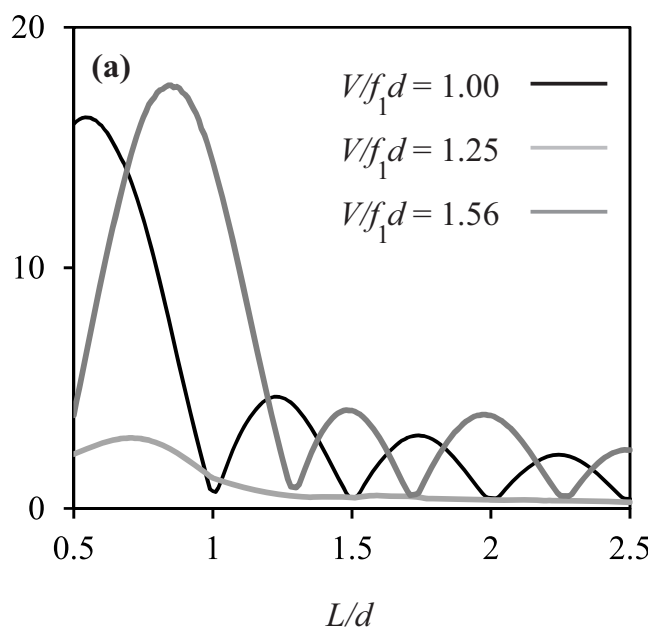

$$
a_{\max }(1.5 L)_{N=6}\left[\mathrm{~m} / \mathrm{s}^{2}\right]
$$

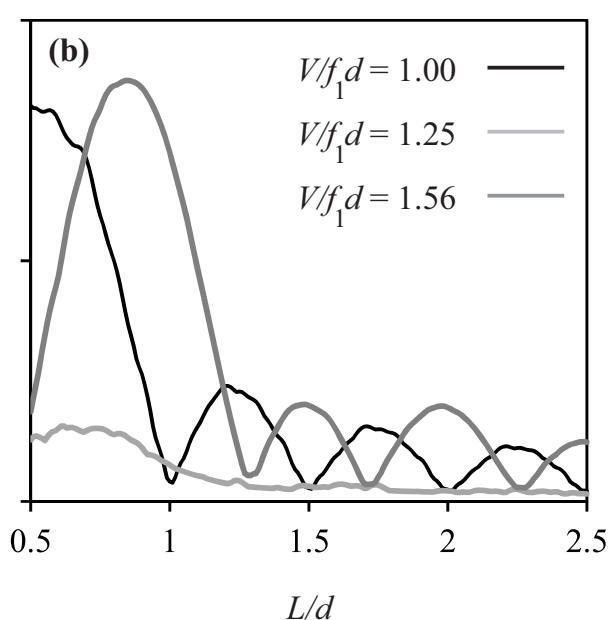

$L / d$

Figure 4: Maximum acceleration at $x=1.5 L$ vs. $L / d$ for $V / f_{1} d=\{1,1.25,1.56\}$. (a) $N=2,($ b) $N=6$. maximum acceleration takes place at the first resonance of the second mode $\left(V / f_{1} d=1.56\right)$ in the vicinity of the first theoretical maximum, i.e. $L / d=0.855$, leading to $17.59 \mathrm{~m} / \mathrm{s}^{2}$ and $17.72 \mathrm{~m} / \mathrm{s}^{2}$ when two and six modes are taken into account, respectively.

Finally, in order to visualise the phenomenon of maximum resonance and cancellation of it, five particular

In order to evaluate the effect of higher modes, the same analysis is performed but including the contribution of the first six modes of vibration, $N=6$, (first three antisymmetric and first three symmetric), and it is represented in Fig. 5. Again, $L / d$ analytical ratios for maximum resonance and cancellation are accurate estimates for real values. The effect of higher modes is visible far from resonance, for moderate values of the response, especially in the acceleration plots. The displacement response is mildly affected by modes higher than $n=2$, which is to be expected and consistent with previous works 21, 22. The overall

$/ d$ ratios are selected, and maximum acceleration envelopes at $x=1.5 L$ are plotted versus the dimensionless velocity of the particular train with characteristic distance $d$ (see Fig. 6). The selected ratios along with the mode number $n$, resonance order $j$ and corresponding maximum or cancellation event $i$ are included in 

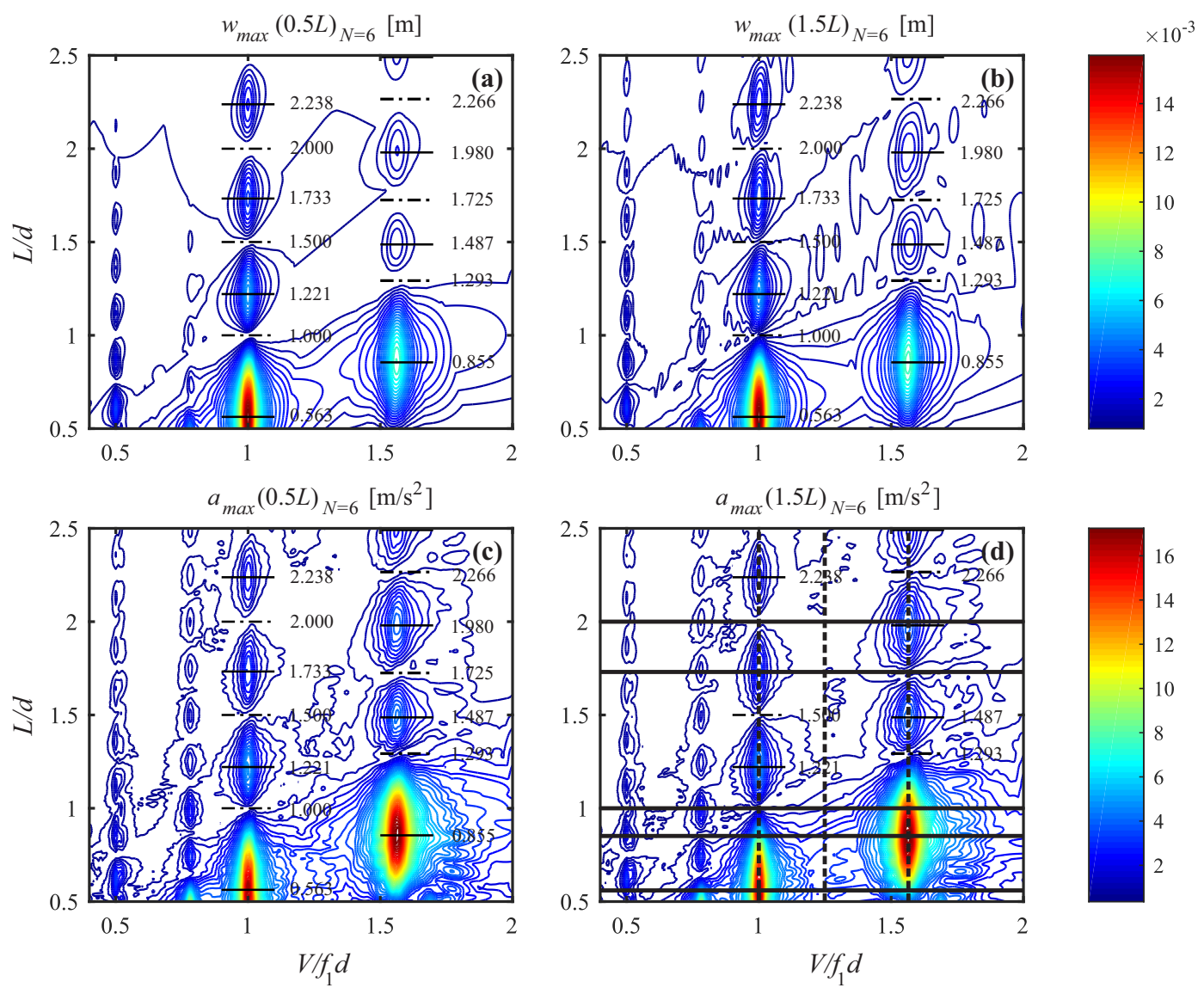

Figure 5: Maximum displacement and acceleration at $x=0.5 L$ and $x=1.5 L$ contour plots for $N=6$.

Table 4. Selected $L / d$ ratios are marked in Fig. 5(d) using a black horizontal continuous trace. The response is calculated considering the contribution of the first six modes of vibration in all the cases. Figs. 6(a) and 6(b) correspond to a train that induces a first maximum resonance of the fundamental mode first resonance; that is, the remaining equidistant trains will also induce this resonance (at different speeds) but leading to lower acceleration amplitudes. Fig. 6(b) represents the time-history for the corresponding resonant velocity marked with a discontinuous vertical trace in Fig. 6(a). Figs. 6(c) and 6(d) represent the acceleration associated to a train with a characteristic distance $d$ such that when inducing a first resonance of the second symmetric mode, this also takes place at the most prominent maximum $(i=1)$. Again, no other train will lead to a higher acceleration response at this particular resonance. Figs. 6(e)-(f) and 6(g)-(h) correspond to the first and second cancellation of the first resonance of the fundamental and second modes first resonance, respectively. It is obvious that the resonant peaks that should appear at $V / f_{1} d=1$ and at 1.56 , respectively, vanish due to the cancellation event in both cases. Finally, Figs. 6(i) and (j) correspond to a train for which, when inducing a first resonance of the fundamental mode, this happens at a third cancellation condition 

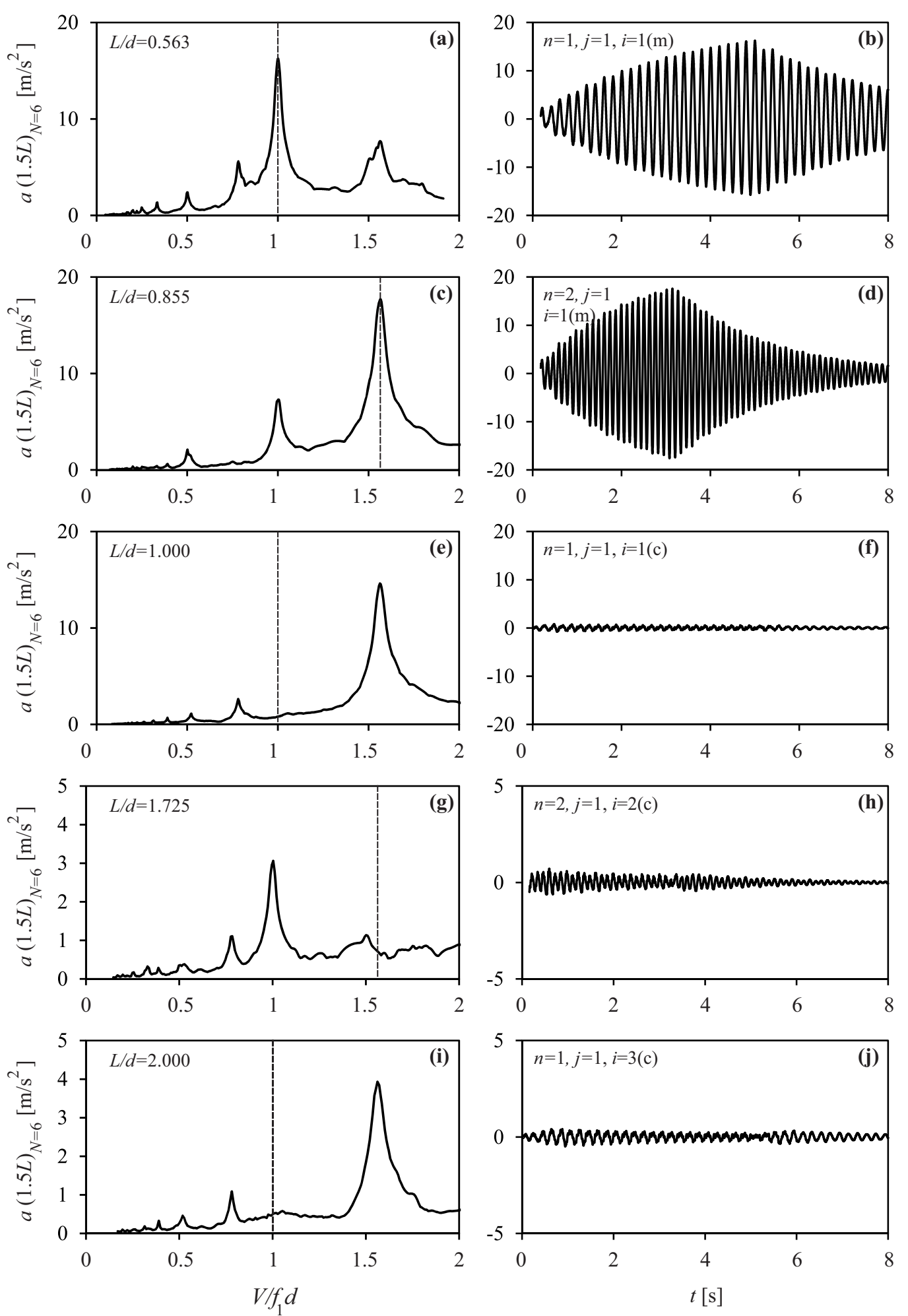

Figure 6: Maximum acceleration and acceleration time history at $x=1.5 L$ vs. $V / f_{1} d$ for different $L / d$ ratios. 
and, therefore, does not take place either. It is important to stress that the response is calculated including the contribution of six modes and in the presence of damping. For these two reasons, the suppression of the response is not complete.

\begin{tabular}{lllllll}
\hline$(L / d)$ & $n$ & $j$ & $i$ & $\mathrm{~m} / \mathrm{c}$ & Event & Fig. 6$]$ \\
\hline 0.563 & 1 & 1 & 1 & $\mathrm{~m}$ & $1^{\text {st }}$ maximum of $1^{\text {st }}$ mode $1^{\text {st }}$ resonance & $(\mathrm{a})-(\mathrm{b})$ \\
0.855 & 2 & 1 & 1 & $\mathrm{~m}$ & $1^{\text {st }}$ maximum of $2^{\text {nd }}$ mode $1^{\text {st }}$ resonance & $(\mathrm{c})$-(d) \\
1.000 & 1 & 1 & 1 & $\mathrm{c}$ & $1^{\text {st }}$ cancellation of $1^{\text {st }}$ mode $1^{\text {st }}$ resonance & $(\mathrm{e})-(\mathrm{f})$ \\
1.725 & 2 & 1 & 2 & $\mathrm{c}$ & $2^{\text {nd }}$ cancellation of $2^{\text {nd }}$ mode $1^{\text {st }}$ resonance & $(\mathrm{g})-(\mathrm{h})$ \\
2.000 & 1 & 1 & 3 & $\mathrm{c}$ & $3^{\text {nd }}$ cancellation of $1^{\text {st }}$ mode $1^{\text {st }}$ resonance & $(\mathrm{i})-(\mathrm{j})$ \\
\hline
\end{tabular}

Table 4: $L / d$ ratios and associated events represented in Fig. 6

From the previous analysis it can be concluded that when resonance is induced on a two-span continuous beam or bridge by a train of equidistant loads, its amplification will depend on the level of free vibrations associated with the particular velocity. Resonances of either the first (antisymmetric) or second (symmetric) mode are prone to be responsible for the overall maximum vertical acceleration of the bridge. The same train will require a higher speed in order to induce the same resonance of the second mode (when compared to that of the fundamental), with a higher level of free vibrations left by each axle load. Therefore, the second mode can be the one responsible for the maximum response as long as the train speed is sufficiently high. In other words, the mode causing the maximum overall response of the bridge will depend on the maximum train speed.

Depending on the ratio between the length and the train characteristic distance, the response at resonance may be rather prominent or almost imperceptible. Furthermore, the analytical predictions of these $L / d$ ratios leading to maximum resonance or cancellation of it, which were obtained in closed form in the absence of damping and admitting separate modal contributions, show themselves to be good estimates of the real values. This is due to the moderate damping values in railway bridges and also to the fact that, at resonance, the contribution of modes other than the one undergoing resonance is very limited.

\section{Case studies}

In the previous section, the conditions for maximum resonance and its cancellation have been analysed in the ideal case of perfectly equidistant trains of loads and unlimited train speeds in order to investigate the evolution of the resonant amplitudes in the complete domain of non-dimensional speeds and $L / d$ ratios. In this section, the analysis of the dynamic performance of two real bridges is presented under the circulation of articulated load trains considering realistic upper-limit design speeds. 
The bridges of interest, described in subsections 4.1 and 4.2 , belong to the Swedish railway network, in particular, to the Bothnia and to the West Coast lines, respectively. The maximum train speed at both sites is at present $200 \mathrm{~km} / \mathrm{h}$. The possibility of increasing the operating speed on these lines with a target of $250 \mathrm{~km} / \mathrm{h}$ is currently under study, and the evaluation of the performance of the bridges if these lines are upgraded is a topic of major interest for the Swedish railway administration [32], 33].

The structures under study are single-track and, in a first approach, the contribution of modes other than the longitudinal bending ones (e.g. torsion, transverse bending) is disregarded. The response of the bridges under the circulation of the High Speed Load Model-A (HSLM-A) from Eurocode [30] (see Appendix A for train model definition) is obtained by time integration, admitting that the total response can be expressed as a combination of the analytical modes of the two-span continuous BE beam as described in section 3 (i.e. the natural frequencies and mode shapes are obtained applying Eqs. 3a, $3 \mathrm{~b}$ and $4 \mathrm{~b}$. The design speed considered for both bridges is $300 \mathrm{~km} / \mathrm{h}$ (i.e. 1.2 times the target operating speed).

\subsection{Case 1. Bridge over River Lögde in Västerbotten, Sweden}

The first structure under study is a bridge crossing the River Lögde on the Bothnia railway line, between the cities of Örnsköldsvik and Gimonäs. It is a continuous bridge with two $43 \mathrm{~m}$ identical spans and a uniform steel-concrete composite deck, as shown in Fig. 7. The deck accommodates a single ballasted track. The main properties of the beam model for this bridge are those also used in 33, and are summarised in Table 8. A modal damping ratio of $0.5 \%$ is admitted as recommended in [30] for composite bridges of the aforementioned span length.
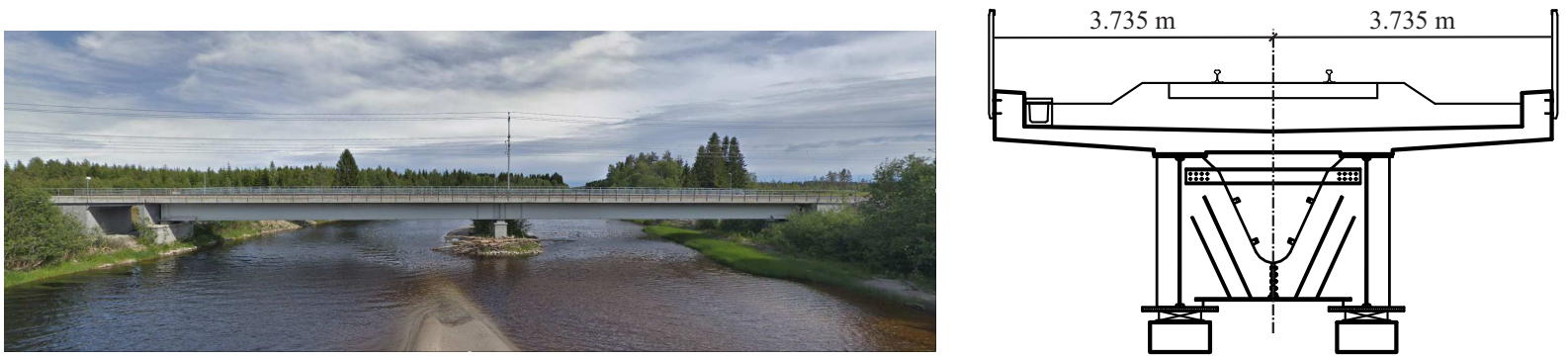

Figure 7: Bridge over the Lögde river. Elevation view and cross-section.

The first two bending frequencies of the bridge are 2.34 and $3.66 \mathrm{~Hz}$. Table 6 presents the critical speeds for the ten HSLM-A trains' characteristic distances for the lowest resonance order attainable given the design speed, and for the first two modes, which will be the ones that contribute most to the acceleration response, as shown later on. From now on, $d_{k}$ stands for the characteristic distance of the $k$-th train. The dimensional resonant speeds are computed applying Eq. 13, and the non-dimensional ones refer to the fundamental mode for both resonant velocities of the first and the second mode, for convenience. All the trains induce first 


\begin{tabular}{lll}
\hline & Lögde bridge & Förslöv bridge \\
\hline$L(\mathrm{~m})$ & 43.00 & 23.50 \\
$E I\left(\mathrm{Nm}^{2}\right)$ & $1.05 \cdot 10^{11}$ & $7.14 \cdot 10^{10}$ \\
$\rho A(\mathrm{~kg} / \mathrm{m})$ & 13816 & 23010 \\
$\zeta_{n}(\%)$ & 0.50 & 1.00 \\
$f_{1}, f_{2}(\mathrm{~Hz})$ & $2.34,3.66$ & $5.01,7.83$ \\
\hline
\end{tabular}

Table 5: Properties of the Lögde and Förslöv bridges. lowest resonance order attainable for this second mode for trains A6 to A10 is then second order $(j=2)$.

\begin{tabular}{llllllll|lllll}
\hline \multirow{2}{*}{$\operatorname{Tr}$} & \multirow{2}{*}{$d_{k}(\mathrm{~m})$} & $L / d_{k}$ & \multicolumn{6}{c|}{$n=1$} & \multicolumn{5}{c}{$n=2$} \\
\cline { 5 - 12 } & & & $j$ & $V_{1 j}^{r}\left(\frac{\mathrm{km}}{h}\right)$ & $K_{1 j}^{r}$ & $R_{1} F_{P k}$ & $R_{1} F_{P k} / \omega_{1}^{2}$ & $j$ & $V_{2 j}^{r}\left(\frac{\mathrm{km}}{h}\right)$ & $K_{1 j}^{r}$ & $R_{2} F_{P k}$ & $R_{2} F_{P k} / \omega_{2}^{2}$ \\
\hline $\mathrm{A} 1$ & 18 & 2.39 & 1 & 151.8 & 0.209 & 0.28 & $1.30 \cdot 10^{-3}$ & 1 & 237.1 & 0.327 & 0.39 & $7.38 \cdot 10^{-4}$ \\
$\mathrm{~A} 2$ & 19 & 2.26 & 1 & 160.2 & 0.221 & 0.54 & $2.52 \cdot 10^{-3}$ & 1 & 250.3 & 0.345 & 0.01 & $2.00 \cdot 10^{-5}$ \\
$\mathrm{~A} 3$ & 20 & 2.15 & 1 & 168.6 & 0.233 & 0.42 & $1.95 \cdot 10^{-3}$ & 1 & 263.5 & 0.363 & 0.48 & $9.05 \cdot 10^{-4}$ \\
$\mathrm{~A} 4$ & 21 & 2.05 & 1 & 177.1 & 0.244 & 0.17 & $7.90 \cdot 10^{-4}$ & 1 & 276.7 & 0.382 & $\mathbf{0 . 8 3}$ & $1.56 \cdot 10^{-3}$ \\
$\mathrm{~A} 5$ & 22 & 1.95 & 1 & 185.5 & 0.256 & 0.15 & $7.12 \cdot 10^{-4}$ & 1 & 289.8 & 0.400 & 0.79 & $1.50 \cdot 10^{-3}$ \\
$\mathrm{~A} 6$ & 23 & 1.87 & 1 & 193.9 & 0.267 & 0.45 & $2.06 \cdot 10^{-3}$ & 2 & 151.5 & 0.209 & 0.01 & $2.20 \cdot 10^{-5}$ \\
$\mathrm{~A} 7$ & 24 & 1.79 & 1 & 202.4 & 0.279 & 0.65 & $3.02 \cdot 10^{-3}$ & 2 & 158.1 & 0.218 & 0.34 & $6.45 \cdot 10^{-4}$ \\
$\mathrm{~A} 8$ & 25 & 1.72 & 1 & 210.8 & 0.291 & 0.70 & $\mathbf{3 . 2 2} \cdot \mathbf{1 0}^{-3}$ & 2 & 164.7 & 0.227 & 0.38 & $7.22 \cdot 10^{-4}$ \\
$\mathrm{~A} 9$ & 26 & 1.65 & 1 & 219.2 & 0.302 & 0.68 & $3.12 \cdot 10^{-3}$ & 2 & 171.3 & 0.236 & 0.15 & $2.89 \cdot 10^{-4}$ \\
$\mathrm{~A} 10$ & 27 & 1.59 & 1 & 227.6 & 0.314 & 0.47 & $2.18 \cdot 10^{-3}$ & 2 & 177.9 & 0.245 & 0.23 & $4.37 \cdot 10^{-4}$ \\
\hline
\end{tabular}

Table 6: Lögde bridge highest attainable resonant speeds and free vibration amplitudes for $n=1,2$ under HSLM-A trains.

In order to compare the level of free vibrations associated to each train at the resonant speeds, the values of $K_{1 j}^{r}$ are superimposed to the normalised amplitude of the free vibrations for the first two modes, $R_{1}$ and $R_{2}$ in Fig. 8. The vertical black lines stand for the resonant speeds of the fundamental mode and the grey vertical ones to those of the second mode. The intersection of each vertical line with either $R_{1}\left(K_{1}\right)$ or $R_{2}\left(K_{1}\right)$ (only intersections of traces of the same colour should be considered) provides an estimation of the level of acceleration experienced by the bridge due to the accumulation of free vibrations in a certain mode at resonance. Moreover, as per Eq. 11, the amplitude of either $R_{1}$ or $R_{2}$ is multiplied by the factor $F_{P k}=P_{k} / P_{1}$ in order to account for the different axle load modulus of the HSLM-A trains, $P_{k}$ being the axle load of the $k$-th train and $P_{1}=170 \mathrm{kN}$, which is the minimum value. This corrected product is shown 
with a red circle that has a black border in the case of resonances of the first antisymmetric mode and a grey border in the case of resonances of the second mode. Admitting that the number of loads is sufficient and that the resonance state has reached a constant amplitude due to the presence of damping, this may be used to compare the relative amplitudes of the acceleration at resonance induced by different trains on the first two modes of vibration. This is of course an estimation that only takes into account the geometry of the compositions (i.e. the lengths of the passengers' coaches) and admits similar modal damping ratios for both modes, but it allows a preliminary prediction of which train will induce the most detrimental resonance and which mode will be the one undergoing it, taking into consideration all cancellation and maximum free vibration situations. In Table 6 the values of $R_{n} F_{P k}$ and $R_{n} F_{P k} / \omega_{n}^{2}$ are included as well for each train and resonance speed. Notice that the first is proportional to the amplitude of the accelerations in free vibration and the latter to the amplitude of the displacements as per Eq. 11. Also, the overall maximum value for each of these ratios is highlighted in bold. According to this, the maximum displacement could occur when train A8 induces first resonance of the fundamental mode $(j=1, n=1)$, while the maximum acceleration may take place at first resonance of the second mode induced by train A4. This train should be one of the most aggressive trains for the particular structure and speed limit according to the Serviceability Limit State for traffic safety.

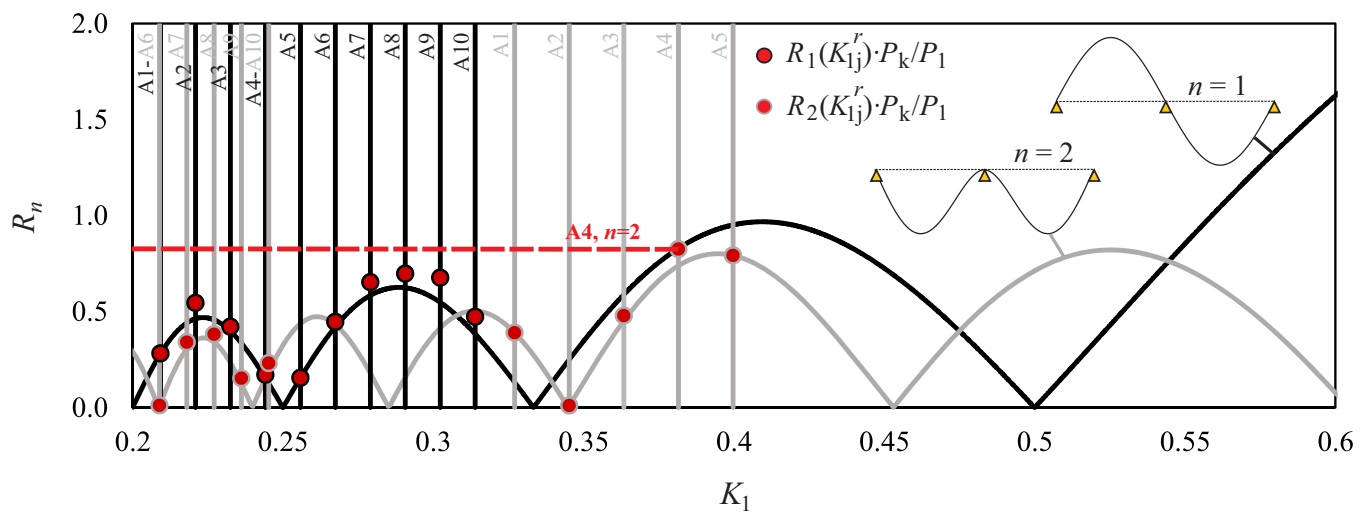

Figure 8: Lögde bridge. $R_{n}$ vs. $K_{1}$ for $n=1,2$ and the most critical resonant non-dimensional speeds from HSLM-A trains under $300 \mathrm{~km} / \mathrm{h}$.

The response of the Lögde bridge to the circulation of the ten HSLM-A trains is now calculated and presented in the range of travelling speeds $[20,83.33] \mathrm{m} / \mathrm{s}$ with $\Delta V=0.5 \mathrm{~m} / \mathrm{s}$ (i.e. $[72,300] \mathrm{km} / \mathrm{h}$ with $\Delta V=1.8 \mathrm{~km} / \mathrm{h})$. The maximum response is obtained considering the contribution of the first two modes and the first six modes at sections $x / L=[0.25,0.5,0.75,1.25,1.5,1.75]$. The overall maximum acceleration takes place at mid-span of the second $\operatorname{span} x / L=1.5$ in both cases. In Fig. 9 the maximum transverse displacement and acceleration are plotted in absolute values at this most critical section versus the non- 
dimensional speed $V / f_{1} d$ for the ten HSLM-A trains. Plots (a) and (b) are calculated taking into account the first two modal contributions $(N=2)$, while plots (c) and (d) represent the response calculated with six modes $(N=6)$. The maximum response induced by the HSLM-A4 is represented with a red trace. The overall maximum acceleration reaches $6.01 \mathrm{~m} / \mathrm{s}^{2}$ for $N=2$, exceeding the limit for ballasted tracks according to standards [23]. Therefore, this bridge may need to be improved in order to allow increased train speeds. These results are consistent with those presented by Andersson [33. As predicted, for the admitted design velocity the maximum response in terms of accelerations is due to a first resonance of the second mode $\left(V / f_{1} d=1.56\right)$, and it is induced by the HSLM-A4 train (red trace). As per the displacement, train A8 (green trace), together with A7 and A9, lead to the maximum displacement at first resonance of the fundamental mode. Notice in Table 6 that the three trains (A7, A8 and A9) present a very similar value of $R_{1} F_{P k} / \omega_{1}^{2}$. In Fig. 9 it can also be observed that train A2 does not induce first resonance of the symmetric mode. For this train $L / d_{k}=2.26$, very close to the theoretical value 2.266 for cancellation of the second mode first resonance (see Table 3). Finally, it should be noted that the effect of modes higher than the second one is very low, especially at resonance. In the displacement response, the difference is almost imperceptible.
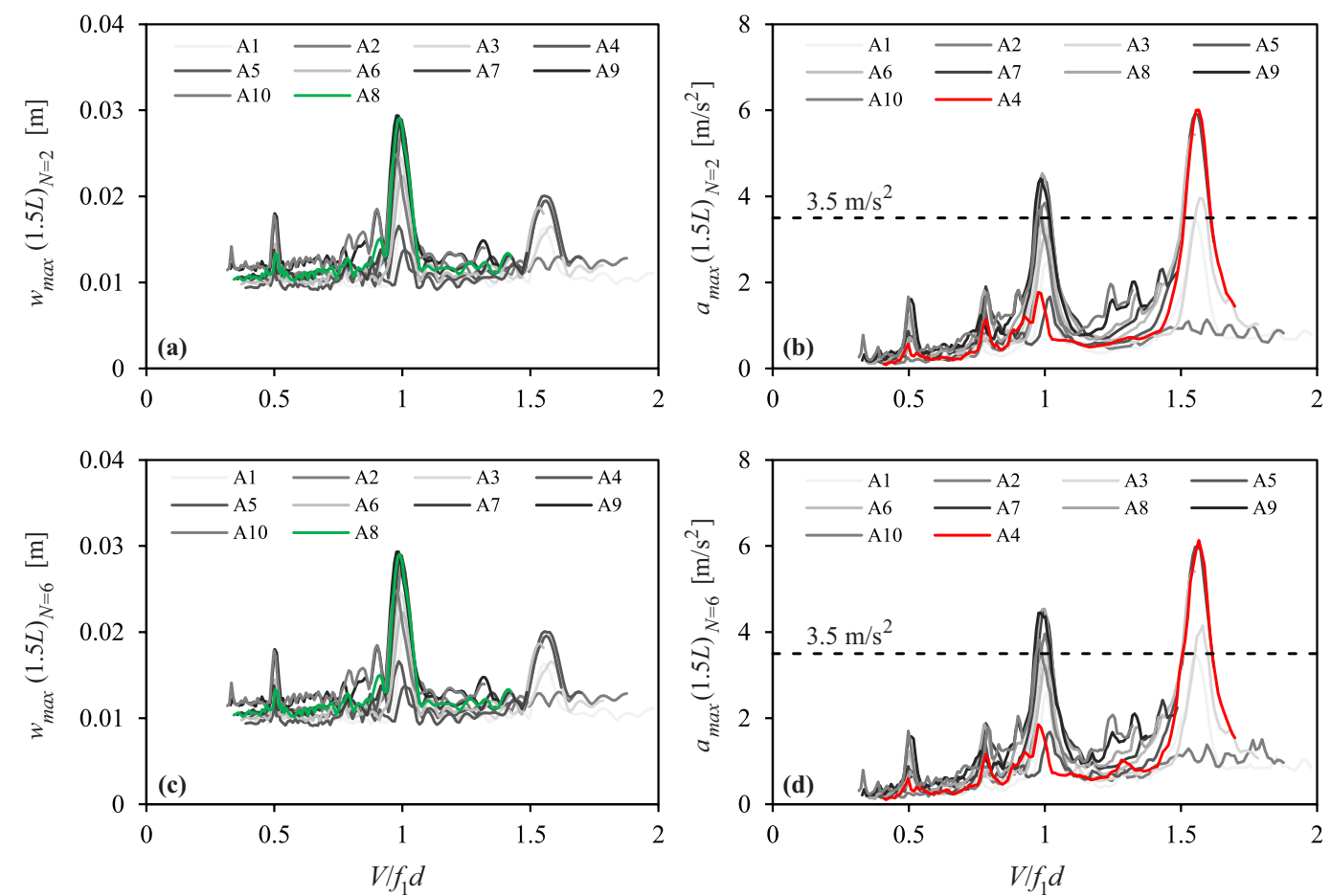

Figure 9: Lögde bridge. (a)-(b) Maximum displacement and acceleration at $x=1.5 L(N=2)$. (c)-(d) Maximum displacement and acceleration at $x=1.5 L(N=6)$. HSLM-A trains, $V_{\max }=300 \mathrm{~km} / \mathrm{h}$. 


\subsection{Case 2. Förslöv bridge in Skåne, Sweden}

As a second example, the case of a pre-stressed concrete railway bridge from the West Coast line located between the cities of Gothenburg and Copenhagen is presented. A modified version of the real structure is analysed, with two identical spans of $23.5 \mathrm{~m}$ and a uniform cross-section with the properties listed in Table 8. The bridge is composed of two structurally independent single-track decks as shown in Fig. 10. A modal damping ratio of $1 \%$ is assigned to each mode as per 30 .
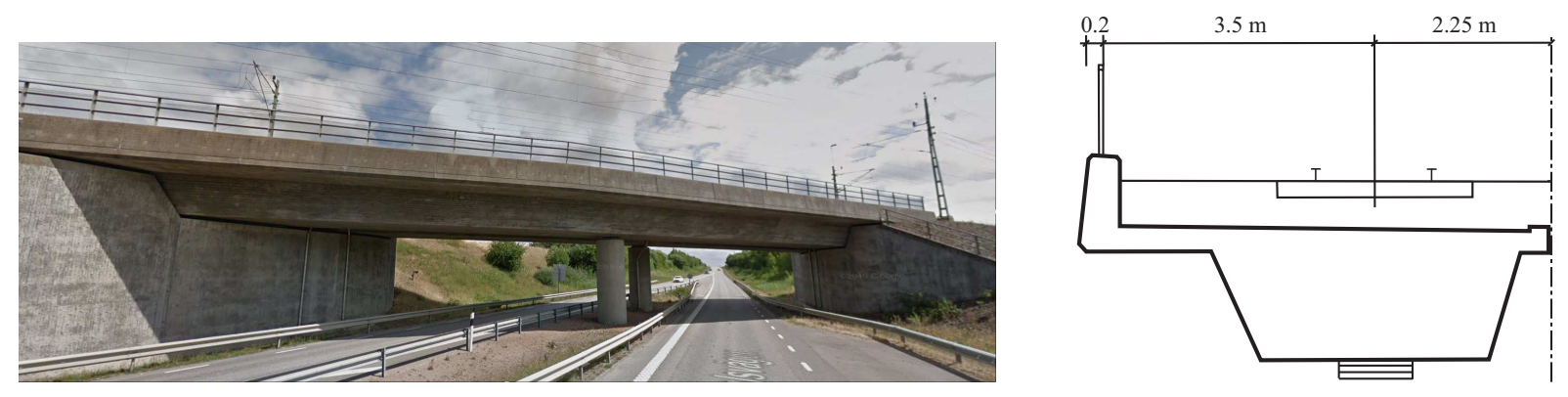

Figure 10: Förslöv bridge. Elevation view and cross-section.

The first two natural frequencies of the bridge calculated analytically are $5.01 \mathrm{~Hz}$ and $7.83 \mathrm{~Hz}$, respectively. Again, the theoretical resonant frequencies are computed for the first two modes and the first resonant orders. In this case study the natural frequencies are higher than in the previous one. For this reason, the critical velocities leading to first resonance of the first two modes exceed the maximum design speed of $300 \mathrm{~km} / \mathrm{h}$ assumed for all the HSLM-A trains. Again, in Table 7 the highest attainable resonant velocities for the first and second modes have been included, along with the ratios $R_{n} F_{P k}$ and $R_{n} F_{P k} / \omega_{n}^{2}$, proportional to the acceleration and displacement amplitudes in free vibration, respectively. The highest values for these two ratios are highlighted in bold. In this case when train A10 induces a second resonance of the fundamental mode, for that particular speed the free vibration amplitudes both for the displacements and the accelerations are maximum. Therefore this train could be one of the most aggressive.

In Fig. 11 the highest attainable non-dimensional resonant speeds for each train are represented with vertical solid traces for modes $n=1$ (black) and $n=2$ (grey). Again, the non-dimensional amplitude of the free vibrations, $R_{n}$ for each train in each mode, is marked with a circle after applying the correcting factor $F_{P k}$. In this case, all the trains in the HSLM model are capable of inducing a second-order resonance of the first antisymmetric mode, but only the first four have a sufficiently low characteristic distance to induce second-order resonance of the first symmetric mode below $300 \mathrm{~km} / \mathrm{h}$. It can be verified graphically that train HSLM-A10 is the one leading to a highest value of $R_{n} F_{P k}$, in particular for the first mode $(n=1)$, as its associated second resonance speed coincides with the third local maximum of the free vibrations for $n=1$.

In what follows, the response of the bridge is obtained numerically under the ten HSLM-A trains. Fig. 


\begin{tabular}{lll|lllll|lllll}
\hline \multirow{2}{*}{$\operatorname{Tr}$} & \multirow{2}{*}{$d_{k}(\mathrm{~m})$} & $L / d_{k}$ & \multicolumn{6}{c|}{$n=1$} & \multicolumn{5}{c}{$n=2$} \\
\cline { 5 - 12 } & & & & $V_{1 j}^{r}\left(\frac{\mathrm{km}}{h}\right)$ & $K_{1 j}^{r}$ & $R_{1} F_{P k}$ & $R_{1} F_{P k} / \omega_{1}^{2}$ & $j$ & $V_{2 j}^{r}\left(\frac{\mathrm{km}}{h}\right)$ & $K_{1 j}^{r}$ & $R_{2} F_{P k}$ & $R_{2} F_{P k} / \omega_{2}^{2}$ \\
\hline $\mathrm{A} 1$ & 18 & 1.31 & 2 & 162.3 & 0.191 & 0.26 & $2.58 \cdot 10^{-4}$ & 2 & 253.7 & 0.299 & 0.36 & $1.49 \cdot 10^{-4}$ \\
$\mathrm{~A} 2$ & 19 & 1.24 & 2 & 171.4 & 0.202 & 0.08 & $8.24 \cdot 10^{-5}$ & 2 & 267.7 & 0.316 & 0.59 & $2.43 \cdot 10^{-4}$ \\
$\mathrm{~A} 3$ & 20 & 1.18 & 2 & 180.4 & 0.213 & 0.38 & $3.85 \cdot 10^{-4}$ & 2 & 281.8 & 0.332 & 0.31 & $1.27 \cdot 10^{-4}$ \\
$\mathrm{~A} 4$ & 21 & 1.12 & 2 & 189.4 & 0.223 & 0.52 & $5.29 \cdot 10^{-4}$ & 2 & 295.9 & 0.349 & 0.12 & $5.00 \cdot 10^{-5}$ \\
$\mathrm{~A} 5$ & 22 & 1.07 & 2 & 198.4 & 0.234 & 0.37 & $3.78 \cdot 10^{-4}$ & 3 & 206.7 & 0.244 & 0.14 & $5.71 \cdot 10^{-5}$ \\
$\mathrm{~A} 6$ & 23 & 1.02 & 2 & 207.4 & 0.245 & 0.15 & $1.50 \cdot 10^{-4}$ & 3 & 216.1 & 0.255 & 0.45 & $1.86 \cdot 10^{-4}$ \\
$\mathrm{~A} 7$ & 24 & 0.98 & 2 & 216.4 & 0.255 & 0.16 & $1.59 \cdot 10^{-4}$ & 3 & 225.5 & 0.266 & 0.50 & $2.08 \cdot 10^{-4}$ \\
$\mathrm{~A} 8$ & 25 & 0.94 & 2 & 225.5 & 0.266 & 0.44 & $4.42 \cdot 10^{-4}$ & 3 & 234.9 & 0.277 & 0.26 & $1.08 \cdot 10^{-4}$ \\
$\mathrm{~A} 9$ & 26 & 0.90 & 2 & 234.5 & 0.277 & 0.69 & $6.98 \cdot 10^{-4}$ & 3 & 244.3 & 0.288 & 0.11 & $4.34 \cdot 10^{-5}$ \\
$\mathrm{~A} 10$ & 27 & 0.87 & 2 & 243.5 & 0.287 & $\mathbf{0 . 7 7}$ & $\mathbf{7 . 7 9} \cdot \mathbf{1 0}^{-4}$ & 3 & 253.7 & 0.299 & 0.44 & $1.84 \cdot 10^{-4}$ \\
\hline
\end{tabular}

Table 7: Förslöv bridge highest attainable resonant speeds and free vibration amplitudes for $n=1,2$ under HSLM-A trains.

12 shows the maximum displacement and acceleration responses of the Förslöv bridge. The response is obtained for the ten HSLM-A trains at sections $x / L=[0.25,0.5,0.75,1.25,1.5,1.75]$. The response of the bridge is evaluated for each train in the range of speeds $[20,83.33] \mathrm{m} / \mathrm{s}$ in speed increments of $\Delta V=0.5 \mathrm{~m} / \mathrm{s}$ (i.e. $[72,300] \mathrm{km} / \mathrm{h}$ and $\Delta V=1.8 \mathrm{~km} / \mathrm{h}$ ). The overall maximum acceleration takes place at the mid-span section of the second span, as in the previous example. In Fig. 12 the maximum transverse displacement and acceleration are plotted in absolute values at this most critical section versus the non-dimensional speed $V / f_{1} d$ for the ten trains. The response in Fig. 12(a) and 12 (b) is calculated taking into account the contribution of the first two modes, while that in Fig. 12 (c) and 12.(d) is computed taking into consideration the contribution of the first six modes of vibration.

The maximum acceleration reaches $2.89 \mathrm{~m} / \mathrm{s}^{2}$ when taking into account six modes $\left(2.86 \mathrm{~m} / \mathrm{s}^{2}\right.$ if only the first two are considered), below the recommended limit for traffic safety on ballasted tracks. The maximum acceleration is associated to a second resonance of the fundamental mode (i.e. $V / f_{1} d_{k}=0.5$ ), as predicted in the previous analysis of the level of free vibrations. The train leading to the maximum acceleration response is A10, with $L / d_{k}=0.87$, close to the theoretical ratio for the maximum second resonance of the fundamental mode (see Table 2) and also consistent with what is shown in Table 7 and Fig. 11. This train is also the one responsible for the maximum displacement, which is also caused by the second resonance of the fundamental mode. Only a few trains are able to induce second resonance of the second mode (i.e. $\left.V / f_{1} d=0.78\right)$ and the response in terms of accelerations is much lower than that induced by the most aggressive train in the first mode. In Fig. 12 it can also be observed that train A2 does not induce second resonance of the fundamental mode. For this particular train and bridge $L / d_{k}=1.24$, very close to the 


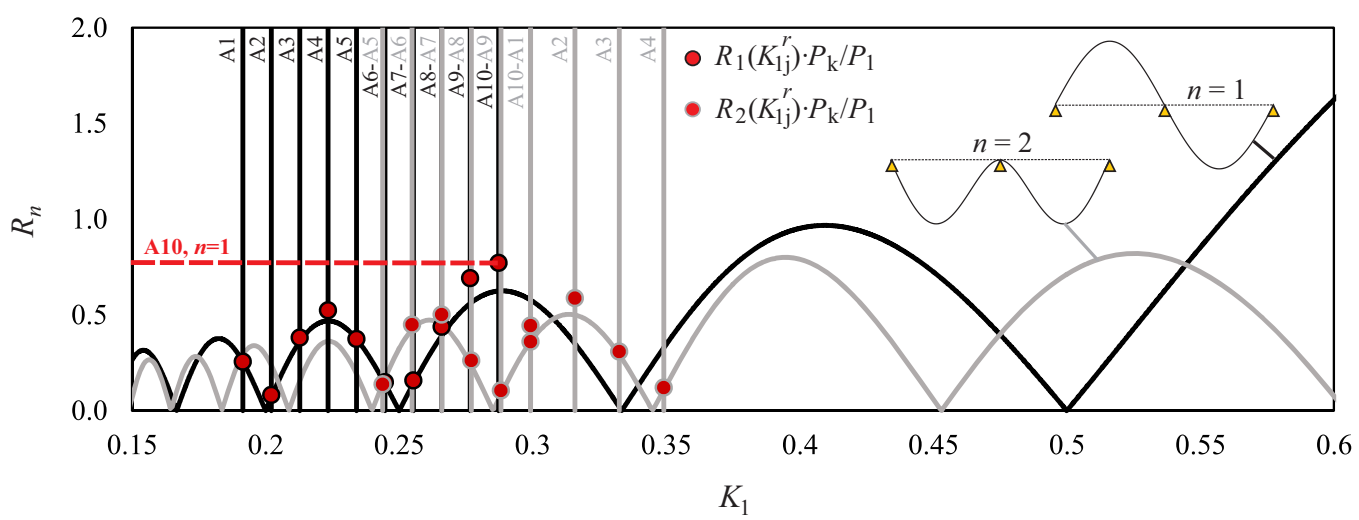

Figure 11: Förslöv bridge. $R_{n}$ vs. $K_{1}$ for $n=1,2$ and the most critical resonant non-dimensional speeds from HSLM-A trains under $300 \mathrm{~km} / \mathrm{h}$.

theoretical value of 1.25 of cancellation of this particular resonance (see Table 2). It is important to state, again, that the maximum response is mainly governed by the first two modes of vibration of the two-span beam, and that the contribution of higher modes in the acceleration response at resonance is negligible.

Finally, in order to summarize the steps applied in this section to propose the particular train leading to the maximum displacement and acceleration of the bridges at resonance a flow chart is included in Fig. 13.

\section{Conclusions}

In this work the vertical response of two-span uniform continuous beams under moving equidistant loads is investigated. The main practical application is to assess the maximum acceleration in railway bridges induced by trains travelling at resonant speeds, and its relation with the level of free vibrations left by each axle load and governed by the existence of cancellation and maximum free vibration phenomena.

First, the problem of a uniform two-span continuous beam is formulated, and a unitless expression for the free vibration amplitudes left by the circulation of a single load travelling at constant speed in a generic mode is obtained in terms of a speed parameter. The existence of cancellation and maximum free vibration conditions is proven, both for symmetric and antisymmetric modes, and the conditions for both situations are presented and analysed. Second, the forced vibrations of the beam under sequences of equidistant trains are analysed numerically, and $L / d$ ratios are derived leading to cancelled or maximum amplitude resonances. Due to the non-dimensional nature of the formulation used, these ratios are applicable to any structure. The above mentioned derivations enable us to understand what type of resonance will lead to the highest acceleration response of the bridge and what type of train may be the most detrimental for a certain design velocity, limiting the analysis to exclusively geometrical considerations. Finally, the previous 

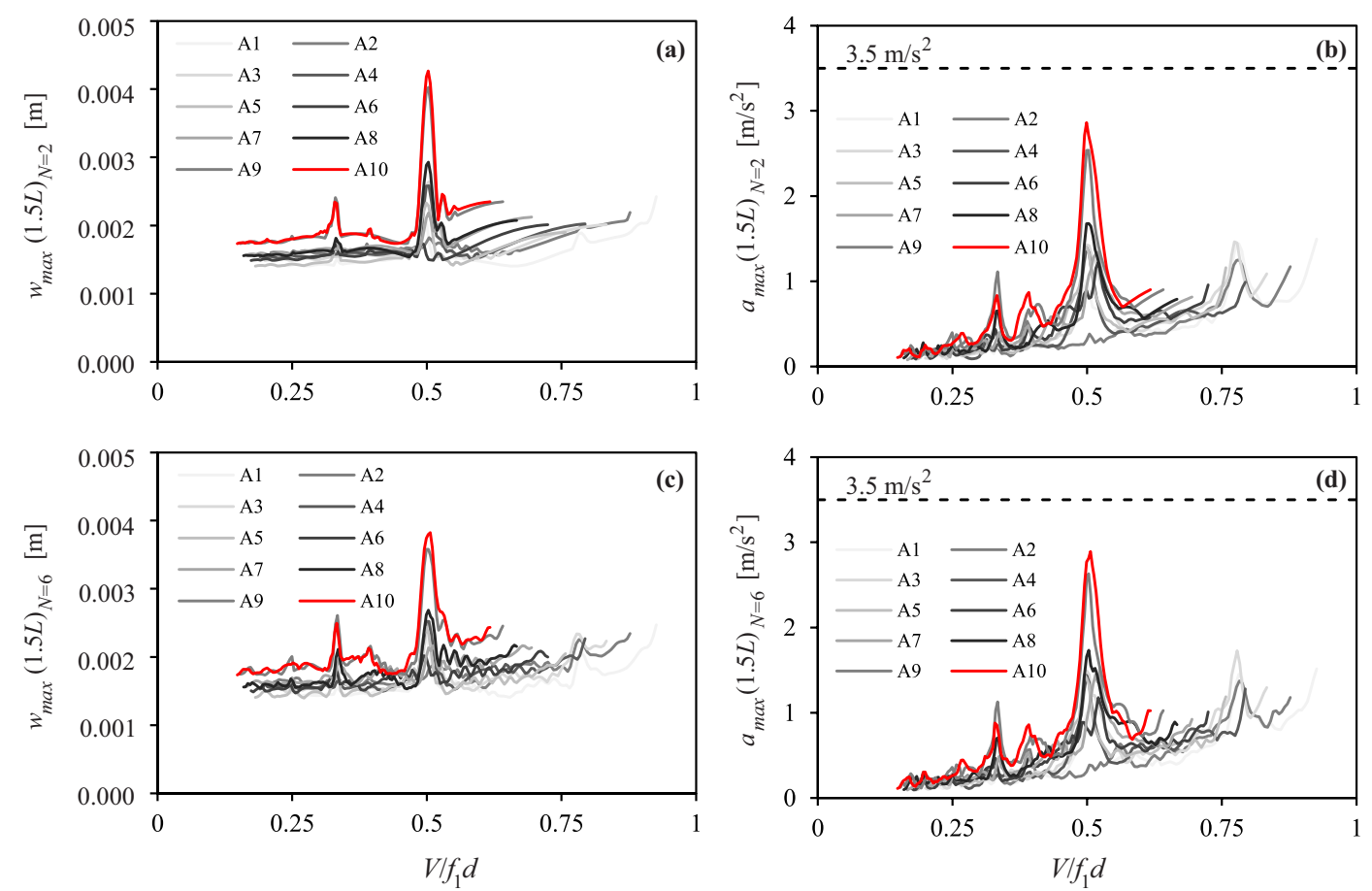

Figure 12: Förslöv bridge. (a)-(b) Maximum displacement and acceleration at $x=1.5 L(N=2)$. (c)-(d) Maximum displacement and acceleration at $x=1.5 L(N=6)$. HSLM-A trains, $V_{\max }=300 \mathrm{~km} / \mathrm{h}$.

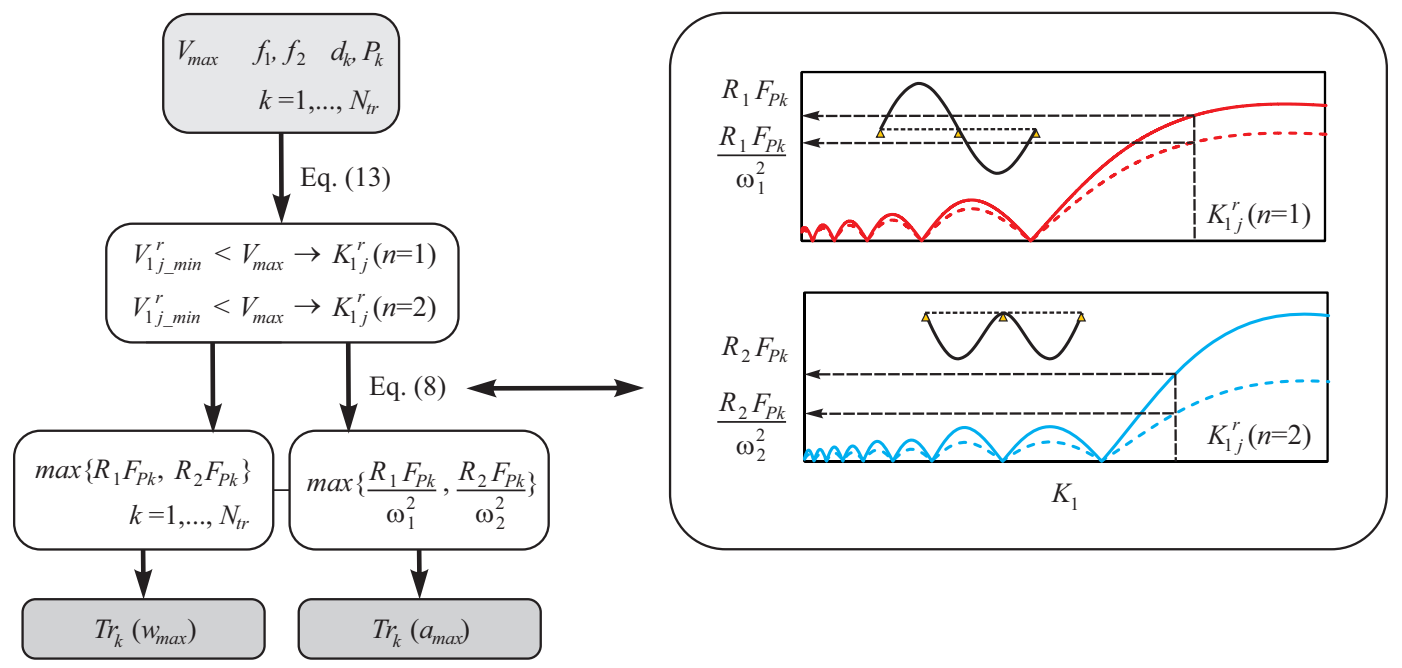

Figure 13: Steps applied to identify trains leading to the maximum displacement and acceleration at resonance. 
theoretical derivations are applied to two case studies of two bridges from the Swedish railway network and their applicability is shown.

The main conclusions derived from the research conducted are:

- When a load moving at constant speed travels on a two-span continuous beam, the level of free vibrations left by the load in a certain mode may be maximum or negligible, depending on the ratio between the load velocity and the beam frequency. These conditions for maximum free vibration and cancellation can be obtained analytically in a non-dimensional format.

- Linear velocities that cancel out the free vibrations in the first antisymmetric mode also cancel out the response of the remaining antisymmetric modes. This does not occur among the symmetric modes or among these and the fundamental one.

- When resonance is induced on a two-span continuous beam or bridge by a train of equidistant loads, its amplification will depend on the level of the free vibrations associated to the particular velocity. Moreover, depending on the ratio between the length and the train characteristic distance, the response at resonance may be rather prominent or almost imperceptible.

- The analytical predictions of the $L / d$ ratios leading to maximum resonance or its cancellation, obtained in the absence of damping and admitting separate modal contributions, show themselves to be excellent estimates of the real values when several modal contributions and modal damping are considered.

- The maximum acceleration response in a two-identical-span railway bridge is mostly governed by the first antisymmetric and first symmetric modes. If a bridge undergoes resonance of the first two modes, the one leading to the maximum acceleration will depend on the maximum design speed. The non-dimensional free vibration amplitudes at the actual resonant speeds may be used to estimate the particular train, resonance order and mode number leading to the overall maximum acceleration response in the structure.

The previous conclusions constitute a theoretical basis limited to the effect of the train axles distribution. Additional phenomena may modify the maximum acceleration response, such as the effects of vehicle-trackbridge or soil-structure interaction, which are beyond the scope of this study.

\section{Appendix A}

Definition of High Speed Load Model-A from Eurocode [30]: 


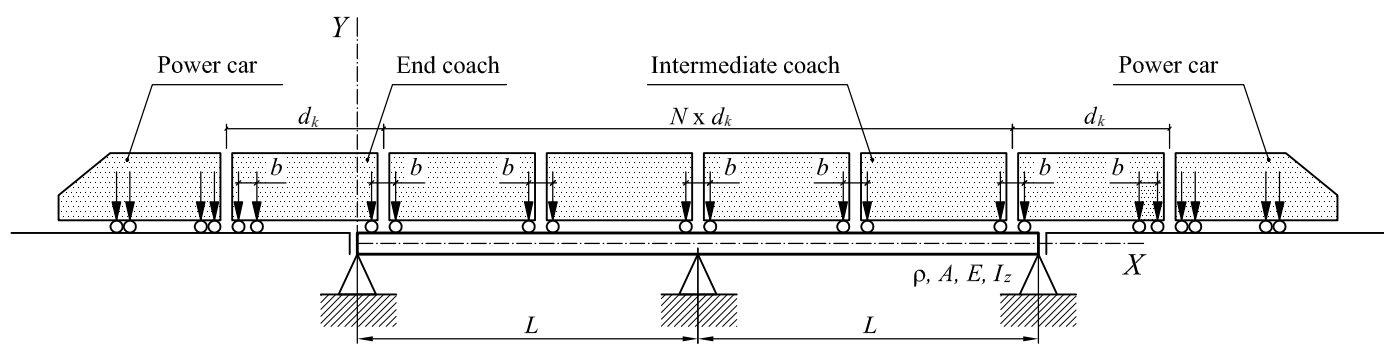

Figure 14: HSLM-A loading scheme.

\begin{tabular}{llllc|lllll}
\hline Train & $N$ & $d_{k}(\mathrm{~m})$ & $b(\mathrm{~m})$ & $P_{k}(\mathrm{kN})$ & Train & $N$ & $d_{k}(\mathrm{~m})$ & $b(\mathrm{~m})$ & $P_{k}(\mathrm{kN})$ \\
\hline A1 & 18 & 18 & 2.0 & 170 & A6 & 13 & 23 & 2.0 & 180 \\
$\mathrm{~A} 2$ & 17 & 19 & 3.5 & 200 & A7 & 13 & 24 & 2.0 & 190 \\
$\mathrm{~A} 3$ & 16 & 20 & 2.0 & 180 & A8 & 12 & 25 & 2.5 & 190 \\
$\mathrm{~A} 4$ & 15 & 21 & 3.0 & 190 & A9 & 11 & 26 & 2.0 & 210 \\
A5 & 14 & 22 & 2.0 & 170 & A10 & 11 & 27 & 2.0 & 210 \\
\hline
\end{tabular}

Table 8: HSLM-A train axle loads $\left(P_{k}\right)$, coach lengths $\left(d_{k}\right)$ and bogie axle spacings $(b)$.

\section{Acknowledgements}

The authors acknowledge the Spanish Ministry of Culture and Sport and the Ministry of Science, Innovation and Universities for the financial support received in the framework of (i) programme José Castillejo with reference number CAS18/00080 for mobility of researchers; and research projects (ii) [BIA2016-75042C2] and [PID2019-109622RB].

\section{References}

[1] ERRI D214, Rail bridges for speeds $>200 \mathrm{~km} / \mathrm{h}$. Final report. Part a. Synthesis of the results of D 214 research, European Rail Research Institute, 1999.

[2] M. Zacher, M. Baeßler, Dynamic behaviour of ballast on railway bridges, in: Taylor \& Francis (Ed.), Dynamics of High-Speed Railway Bridges, no. 978-0-203-89540-5, 2008, pp. 99-112.

[3] W. Hoorpah, Dynamic calculations of high-speed railway bridges in France - some case studies, in: Taylor \& Francis (Ed.), Dynamics of High-Speed Railway Bridges, no. 978-0-203-89540-5, 2008, pp. 133-146.

[4] M. Astiz, J. Manterola, A. Martínez, High-speed bridges [Puentes de ferrocarril de Alta Velocidad. In Spanish], Revista de Obras Públicas 3386 (1999) 43-77.

[ [5] I. Bisús, Viaduct typologies in spanish high-speed railway lines [Tipología de viaductos en las líneas de Alta Velocidad en espaa (in Spanish)], Master Thesis, E.T.S. Ingenieros de Caminos, Canales y Puertos de Barcelona, Universitat Politecnica de Catalunya (2010).

[6] Y. Wang, Q. Wei, J. Yau, Interaction response of train loads moving over a two-span continuous beam, International Journal of Structural Stability and Dynamics 13 (1) (2013) 1-18. 
[7] J. Yau, Resonance of continuous bridges due to high-speed trains, Journal of Marine Science and Technology 9 (1) (2001) $14-20$.

[8] Y. Yang, J. Yau, L. Hsu, Vibration of simple beams due to trains moving at high speeds, Engineering Structures 19(11) (1997) 936-944.

[9] Y. Yang, J. Yau, Y. Wu, Vehicle-Bridge Interaction Dynamics: With Applications to High-Speed Railways, World Scientific, 2004.

[10] E. Savin, Dynamic amplification factor and response spectrum for the evaluation of vibrations of beams under successive moving loads, Journal of Sound and Vibration 248 (2) (2001) $267-288$.

[11] P. Museros, E. Moliner, M. Martínez-Rodrigo, Free vibrations of simply-supported beam bridges under moving loads: Maximum resonance, cancellation and resonant vertical acceleration, Journal of Sound and Vibration 332 (2) (2013) 326 -345 .

[12] C. S. Kumar, C. Sujatha, K. Shankar, Vibration of simply supported beams under a single moving load: A detailed study of cancellation phenomenon, International Journal of Mechanical Sciences 99 (2015) 40-47.

[13] R. Ayre, G. Ford, L. Jacobsen, Transverse vibration of a two-span beam under the action of a moving constant force, Journal of Applied Mechanics 17 (1) (1950) 1-12.

[14] L. Frýba, Dynamics of Railway Bridges, Thomas Telford, 1996.

[15] D. Zheng, Y. Cheung, F. Au, Y. Cheung, Vibration of multi-span non-uniform beams under moving loads by using modified beam vibration functions, Journal of Sound and Vibration 212 (1998) 455-467.

[16] Y. Cheung, F. Au, D. Zheng, Y. C. YS, Vibration of multi-span non-uniform bridges under moving vehicles and trains by using modified beam vibration functions, Journal of Sound and Vibration 228 (3) (1999) 611-628.

[17] Y. Dugush, M. Eisenberger, Vibrations of non-uniform continuous beams under moving loads, Journal of Sound and Vibration 254 (5) (2002) 911-926.

[18] C. Johansson, C. Pacoste, R. Karoumi, Closed-form solution for the mode superposition analysis of the vibration in multi-span beam bridges caused by concentrated moving loads, Computers and Structures 119 (2013) 85-94.

[19] J. Kwark, E. Choi, Y. Kim, B. Kim, S. Kim, Dynamic behaviour of two-span continuous concrete bridges under moving high-speed train, Computers and Structures 82 (2004) 463-474.

[20] Y. Wang, Q. Wei, J. Shi, X. Long, Resonance characteristics of two-span continuous beam under moving high speed trains, Latin American Journal of Solids and Structures 7 (2010) 185-199.

[21] J. Yau, Y. Yang, Vertical accelerations of simple beams due to successive loads traveling at resonant speeds, Journal of Sound and Vibration 289 (2006) $210-228$.

[22] J. Yau, Response of a train moving on multi-span railway bridges undergoing ground settlement, Engineering Structures 31 (9) (2009) 2115-2122.

[23] European Committee for Standardization, Eurocode - Basis of structural design. Annex A2: application for bridges, CEN, Brussels, 2002.

[24] A. Doménech, P. Museros, M. Martínez-Rodrigo, Influence of the vehicle model on the prediction of the maximum bending response of simply-supported bridges under high-speed railway traffic, Engineering Structures 72 (2014) 123-139.

[25] T. Arvidsson, R. Karoumi, C. Pacoste, Statistical screening of modelling alternatives in train-bridge interaction systems, Engineering Structures 59 (2014) 693-701.

[26] Y. Yang, M. Cheng, K. Chang, Frequency variations in vehicle-bridge interaction systems, International Journal of Structural Stability and Dynamics 13 (2) (2013) 1350019.

[27] M. Martínez-Rodrigo, P-Galvín, A. Doménech, A. Romero, Effect of soil properties on the dynamic response of simplysupported bridges under railway traffic through coupled boundary element-finite element analyses, Engineering Structures 170 (2018) 78-90. 
[28] A. Zangeneh, J. Battini, C. Pacoste, R. Karoumi, Fundamental modal properties of simply supported railway bridges considering soil-structure interaction effects, Soil Dynamics and Earthquake Engineering 121 (2019) 212-218.

[29] S. Rao, Vibration of continuous systems, John Wiley \& Sons, 2007.

[30] European Committee for Standardization, Eurocode 1 - Actions on Structures - Part 2: Traffic loads on bridges, CEN, Brussels, 2003.

[31] N. M. Newmark, A method of computation for structural dynamics, Journal of the Engineering Mechanics Division 85 (3) (1959) 67-94.

[32] C. P. C. Johansson, N.A. Nuallin, A. Andersson, A methodology for the preliminary assessment of existing railway bridges for high-speed traffic, Engineering Structures 58 (1).

[33] A. Andersson, Simplified approach to dynamic analysis of railway bridges for high-speed trains, Tech. Rep. Report No. 1837, KTH Royal Institute of Technology, Stockholm (2018). 